\title{
Challenges Facing Percutaneous Ablation in the Treatment of Hepatocellular Carcinoma: Extension of Ablation Criteria
}

\author{
Yanzhao Zhou $\mathbb{1}^{1, *}$ \\ Yi Yang $\left(\mathbb{D}^{2, *}\right.$ \\ Bingyan Zhou ${ }^{3}$ \\ Zhengzheng Wang' \\ Ruili Zhu' \\ Xun Chen' \\ Jingzhong Ouyang' \\ Qingjun Li (D) \\ Jinxue Zhou'
}

\begin{abstract}
'Department of Hepatobiliary and Pancreatic Surgery, Affiliated Cancer Hospital of Zhengzhou University,

Zhengzhou, Henan Province, 450008, People's Republic of China; ${ }^{2}$ Department of Interventional Therapy, National Cancer Center/National Clinical Research Center for Cancer/Cancer Hospital, Chinese Academy of Medical Sciences and Peking Union Medical College, Beijing, I0002I, People's Republic of China; ${ }^{3}$ School of Basic Medical Sciences, Zhengzhou University, Zhengzhou, Henan Province, 45000I,

People's Republic of China
\end{abstract}

*These authors contributed equally to this work

Correspondence: Jinxue Zhou Department of Hepatobiliary and Pancreatic Surgery, Affiliated Cancer Hospital of Zhengzhou University,

Zhengzhou, Henan Province, 450008,

People's Republic of China

Email zhoujx888@I26.com

\begin{abstract}
As an emerging minimally invasive treatment method, percutaneous ablation is more and more widely used in the treatment of liver tumors. It has been recommended by guidelines for diagnosis and treatment of hepatocellular carcinoma (HCC) as a curative treatment alongside surgical resection and liver transplantation. In recent years, with the continuous advancement and innovation of percutaneous ablation technologies, their clinical efficacy and safety have been significantly improved, which has led to the expanded application of percutaneous ablation in the treatment of $\mathrm{HCC}$-more and more patients who were previously considered unsuitable for ablation therapies are now being treated with percutaneous ablation. Obviously, percutaneous ablation can reduce the risk of treatment changes from curative strategies to palliative strategies. Based on clinical practice experience, this review enumerates the advantages and disadvantages of different ablative modalities and summarizes the existing combinations of ablation techniques, thus will help clinicians choose the most appropriate ablative modality for each patient and will provide scientific guidance for improving prognosis and making evidence-based treatment decisions. In addition, we point out the challenges and future prospects of the ablation therapies, thereby providing direction for future research.
\end{abstract}

Keywords: percutaneous ablation, hepatocellular carcinoma, assistive technology, image guidance

\section{Introduction}

In the past two decades, many cohort investigations and comparative studies have shown that the application of percutaneous ablation in the treatment of early-stage hepatocellular carcinoma (HCC) can achieve comparable survival benefits with surgical resection. ${ }^{1-5}$ Based on the accumulated clinical experience and scientific evidence, current HCC diagnosis and treatment guidelines formulated by major academic groups propose percutaneous ablation as a curative treatment for earlystage HCC, especially for patients who cannot undergo liver transplantation due to insufficient liver organ reserves and those who cannot undergo surgical resection. ${ }^{6-10}$ However, studies have shown that up to $36 \%$ of early-stage HCC patients had already received palliative cares [primarily transarterial chemoembolization (TACE)], and thus could not follow the guidelines' recommendations to receive curative treatments such as percutaneous ablation, liver resection or liver transplantation. ${ }^{11,12}$ In addition, ablative modalities have certain limitations. For example, because target tumors cannot be clearly displayed under image guidance, 
as well as many other factors such as tumor location, tumor size, tumor stage, patient status, and complications, around $30 \%$ of early-stage HCC patients were considered unsuitable even for the most mature and effective radiofrequency ablation (RFA) treatment. ${ }^{13,14}$ However, in recent years, with the continuous progress and innovation of percutaneous ablation technologies, more and more patients who were previously considered unsuitable for ablation treatments are receiving percutaneous ablation. ${ }^{15,16}$ Obviously, percutaneous ablation can reduce the risk of palliative cares, ${ }^{17,18}$ which are associated with poor patient prognosis. ${ }^{7,19-21}$ Therefore, when conditions permit, all potential curative treatments for HCC (such as percutaneous ablation, surgical resection, and liver transplantation) should be used as first-line treatments. ${ }^{22}$

The principle of percutaneous ablation is to target and locate tumors under the guidance of imaging technologies, and use physical or chemical methods to kill tumor cells. The imaging techniques include ultrasound, computerized tomography (CT), and magnetic resonance imaging (MRI), while the treatment methods include percutaneous, laparoscopic, and conventional surgery (Figure 1). Percutaneous ablation can directly target tumors and therefore has the advantages of high efficiency and short operation time. In addition, the ablation zone is limited to tumors and their surrounding tissues, which will cause little impact on the body and can be used repeatedly. ${ }^{23-26}$ In the past two decades, percutaneous ablation has developed rapidly, with substantial improvements in both the technologies and procedures. As a result, larger ablation zones can be achieved. This obviously helps to improve the clinical outcomes and safety of the treatment methods, which in turn leads to the expanded application of percutaneous ablation in the treatment of HCC. ${ }^{18,27-29}$ It is challenging, but also necessary, to keep abreast of these developments. The correct use of assistive technologies, equipment and image guidance methods under different ablative modalities will be essential for the optimal treatment results. Therefore, a thorough understanding of indications and contraindications of ablative modalities and familiarity with the advantages and disadvantages of assistive technologies, ablation equipment, and image guidance techniques are of great significance. In addition, tailoring different percutaneous ablation treatment plans for different patients according to the size, location, and histological characteristics of tumors is another key to optimizing clinical results, ${ }^{30}$ which is also in line with the current concept of personalized and precise treatment. ${ }^{31,32}$ To help clinicians choose the most appropriate ablative modality for each patient, we herein have summarized the current clinical practice experience and revealed that more research is required to satisfy the unmet needs of the field.

\section{Understand the Indications and Contraindications for Percutaneous Ablation}

Selecting patients who meet the treatment standards is the key to the success of any ablation or image-guided ablation therapy. The indications and contraindications for percutaneous ablation in the existing clinical practice guidelines should be followed as much as possible. According to the 2018 Barcelona Clinic Liver Cancer (BCLC) staging system and treatment strategy, ablation is suitable for very-earlystage HCC patients with tumor diameter $<2 \mathrm{~cm}$ or those with tumor number $\leq 3$ and single tumor diameter $\leq 3 \mathrm{~cm}^{7}$ However, according to a large number of prospective and retrospective studies and clinical application results published in recent years, the application of percutaneous ablation in the treatment of $\mathrm{HCC}$ has exceeded the recommendations of Barcelona Clinic Liver Cancer (BCLC) staging system and treatment strategy, and has also achieved effective local tumor control. ${ }^{18,20-22,27,28,30}$ By analyzing the clinical research data obtained in recent years, we revised the indications for ablative modalities in the treatment of HCC and divided them into "absolute indications" and "relative indications". "Absolute indications" are HCC cases recommended to be treated with percutaneous ablation in the guidelines. Percutaneous ablation can achieve same curative effects as surgical resection in the treatment of these HCC cases. After sufficient technical training, beginners can completely rely on image-guided percutaneous ablation to successfully treat these cases. "Relative indications" include HCC cases with tumor diameter $\geq 3 \mathrm{~cm}$ or tumor number $\geq 3$ that were recommended to receive TACE treatment by the guidelines, and HCC cases with tumor locations previously considered unsuitable for percutaneous ablation treatments (such as tumors close to the pericardium, diaphragm, gallbladder, caudate lobe of liver, central bile duct, large vessels, lymph node metastasis of HCC, or porta hepatis). ${ }^{18,27,28,33-35}$ These cases were previously considered to be the "forbidden zone", "non-standard indications", or HCC cases with "highrisk locations" for ablative modalities. Safe and effective treatment of these cases requires a wealth of experience in ablation therapy and the use of advanced image guidance technologies (such as fusion imaging and enhanced 


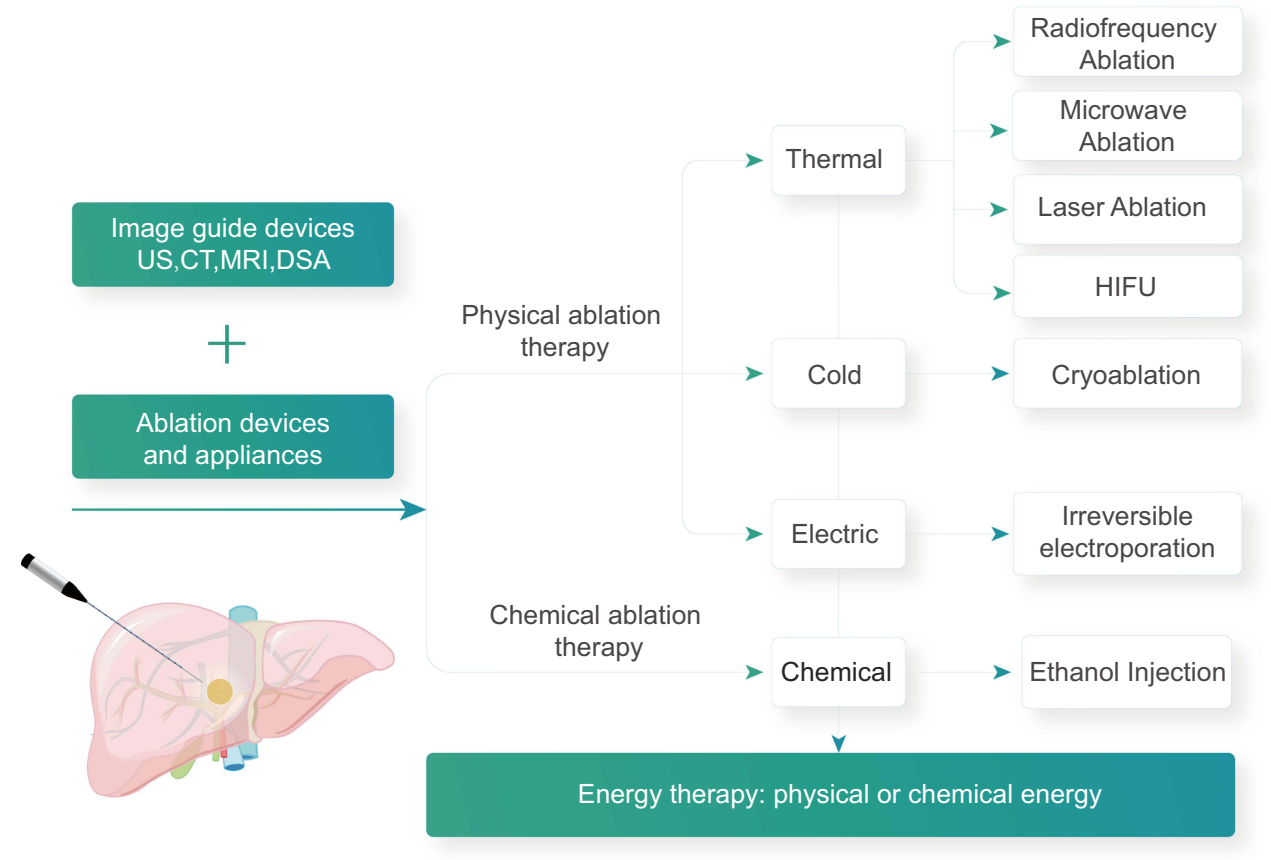

Figure I Percutaneous ablation modalities.

ultrasound) and assistive technologies (such as artificial ascites, artificial hydrothorax, and multiple overlapping ablations).

\section{Assistive Technologies}

Accurate and complete ablation with the help of assistive technologies is essential to obtain the best therapeutic effects. Therefore, many innovations and improvements have been continuously introduced into assistive technologies to improve the curative effects of percutaneous ablation. Superficial HCC tumors have long been considered contraindications for percutaneous ablation, because it is believed that percutaneous ablation can lead to high risks of bleeding, tumor implantation, ${ }^{36}$ and damages to adjacent critical structures [such as diaphragm, ${ }^{37}$ digestive tract and gallbladder, sub-pericardium, and liver sub-capsule]. However, the experience gained from the most commonly used thermal ablation techniques [including RFA and microwave ablation (MWA)] in the past two decades shows that, from the perspective of the risks of bleeding and tumor implantation, subcapsular tumors can be treated safely and effectively with the assist of no-tumor liver parenchyma puncturing, ${ }^{38,39}$ artificial ascites, ${ }^{40,41}$ and artificial hydrothorax techniques. ${ }^{42}$ The principle of artificial ascites technique $^{43}$ is to inject normal saline or isotonic glucose solution into the abdominal cavity through a syringe or a water injection catheter, so that a large water barrier is formed around the liver, thereby separating target tumors from key adjacent organs and effectively preventing the thermal damage caused by ablation (Figure 2). In addition, the leverage effect can also be utilized - by lifting or prying up the tail of the applicator, target tumors can be dragged away from other organs around the liver, thereby further

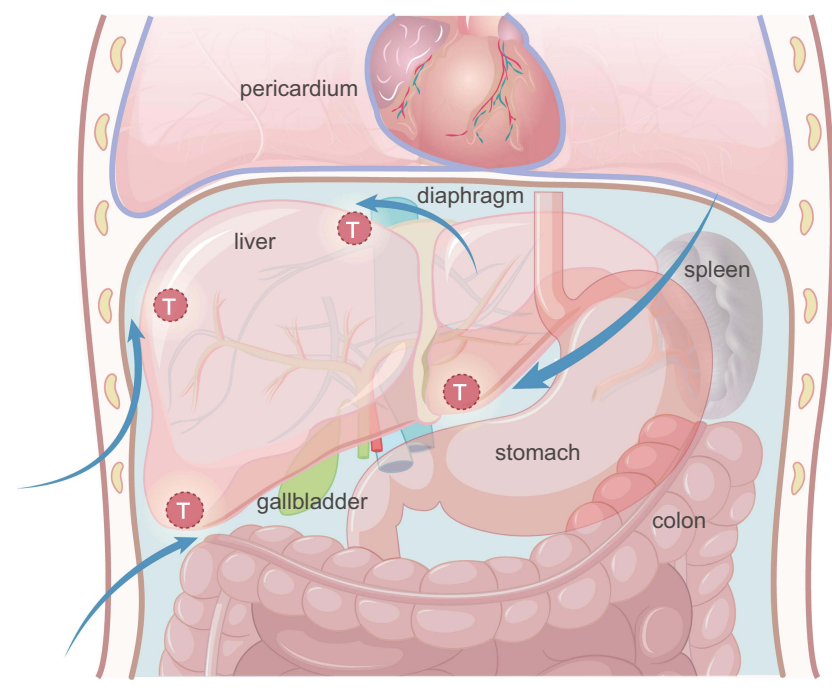

Figure 2 The schematic shows different routes of infusion of the artificial ascites. Depending on the location of the liver tumor, perihepatic, sub-hepatic, subdiaphragm, and gastrohepatic routes can be selected for artificial ascites infusion. A large water barrier is artificially formed to separate the target tumors from key adjacent organs and effectively preventing the thermal damage caused by ablation. 
reducing the thermal damage caused by ablation ${ }^{44}$ (Figure 3 ). Another advantage of artificial ascites technique is that, for patients with a history of abdominal surgery, after artificial ascites is introduced by abdominal catheterization, the adhesive part of the abdominal cavity is also likely to be separated. $^{45}$ However, before choosing percutaneous ablation to treat subcapsular HCC, the latest advances in laparoscopic liver surgery should be fully considered, because surgical resection is currently a safer treatment. ${ }^{27}$

Centrally located HCC cases with tumors close to the main bile duct are still considered to be clear contraindications for thermal ablation techniques such as RFA and MWA, because percutaneous ablation can significantly increase the risk of thermal injury to the biliary tract. ${ }^{46}$ This problem can be solved by performing percutaneous transhepatic bile duct intubation or endoscopic nasobiliary drainage. Both of them can introduce a drainage tube in the bile duct. Intermittently injecting glucose solution with a temperature around $4^{\circ} \mathrm{C}$ into the drainage tube can effectively protect the bile duct from thermal damage caused by $\mathrm{RFA}^{47,48}$ (Figure 3). By performing percutaneous transhepatic gallbladder drainage, saline can be injected into the gallbladder through the drainage tube after the bile is drawn out, ${ }^{49}$ thereby effectively protecting the gallbladder from thermal damage. Alternatively, injecting saline directly into the gallbladder bed and performing RFA after separating the gallbladder and liver capsule (Figure 3) will also reduce the risk of thermal damage to the gallbladder wall. ${ }^{50}$ These assistive technologies are mostly used for percutaneous ablation treatment of large HCC tumors adjacent to the gallbladder.

Different ablation modes will also lead to different clinical outcomes. Monopolar ablation is simple to operate in clinical practice, and ablation can be achieved through a single applicator. Compared with monopolar ablation, multi-bipolar ablation can better predict the ablative margin and expand the ablation zone. ${ }^{51}$ Therefore, the indications for multi-bipolar ablation can be extended to tumors with larger diameters (up to $8 \mathrm{~cm}$ ), even invasive tumors with limited portal vein invasion. ${ }^{52}$ For standard indications (tumor diameter $<5 \mathrm{~cm}$ ), multi-bipolar ablation can also be adopted, especially the no-touch multi-bipolar RFA, which inserts applicators into normal liver tissue around target tumors. Compared with monopolar RFA, notouch multi-bipolar RFA can significantly reduce the local recurrence rate of $\mathrm{HCC}$ with tumor diameter less than $5 \mathrm{~cm}$ (including $\leq 3 \mathrm{~cm}$ ). ${ }^{53}$ In addition, no-touch ablation can safely and effectively ablate various subcapsular and extrahepatic growing HCC tumors ${ }^{54}$ (Figure 4).

\section{Ablation Devices}

Different ablation devices can achieve very similar therapeutic effects (Table 1), but their technical principles and clinical applications are different (Figure 5). Understanding these differences and being familiar with the advantages and limitations of each device will help to choose the most appropriate ablative modality for a patient (Table 2). After all, there is no perfect multifunctional ablation device. In addition, the selection of equipment also depends on the treatment goals and a patient's clinical conditions. ${ }^{16,55-57}$

\section{Radiofrequency Ablation}

Radiofrequency ablation can achieve a complete ablation rate of $90-99 \%$ for tumors with a diameter of $\leq 5 \mathrm{~cm}$ and the highest ablation rate for tumors with a diameter of $\leq 3 \mathrm{~cm} .{ }^{13,58-63}$ As the target tumor size increases, the complete ablation rate decreases. ${ }^{64}$ This is because that when implementing RFA, as the distance between the applicators increases, the heat generated will drop dramatically. In addition to tumor size, the distance between the target tumor and large blood vessels (vessels with a diameter $\geq 3 \mathrm{~mm}$ ) will also affect the complete ablation rate. This is because the blood flow in these large blood vessels will carry heat away from the target tumor. This phenomenon is called the "heat sink effect". ${ }^{65}$ Some current strategies can successfully reduce perfusion-mediated tissue cooling during thermal ablation. One of these strategies is Pringle maneuver, which can cause temporary blood vessel occlusion. ${ }^{66}$ However, this method requires a laparotomy, which obviously makes percutaneous ablation lose the important advantage of minimally invasive. Selective endovascular balloon-occlusion or injection of embolic agents (such as gelatin sponge particles) can also be considered for treatment of difficult cases. ${ }^{67}$ However, the implementation of these strategies requires extra technical experience and extended operation time.

Based on the idea of improving the distribution and depth of energy deposition, ${ }^{68}$ there are currently a variety of designs of radiofrequency electrodes. In addition, radiofrequency generators can transmit radiofrequency energy of various amplitudes and frequencies through applying different pulsing algorithms, ${ }^{69}$ using multiple-electrode switching systems ${ }^{70}$ and adopting different power modes. ${ }^{71}$ Common radiofrequency electrodes used in clinical practice include cluster electrodes, ${ }^{72}$ perfusion electrodes, ${ }^{73}$ internally cooled electrodes, ${ }^{74}$ expandable electrodes, ${ }^{75}$ and adjustable ablation electrodes ${ }^{76}$ (Figure 4). The main purpose of designing these different electrodes is to expand the zone of 
A

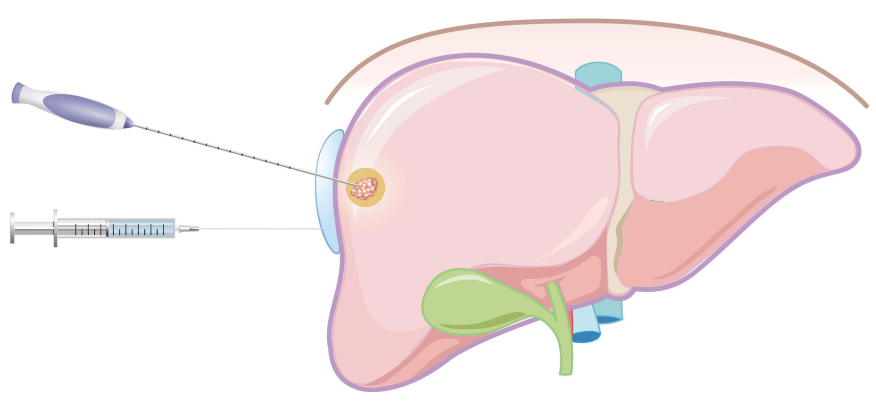

B

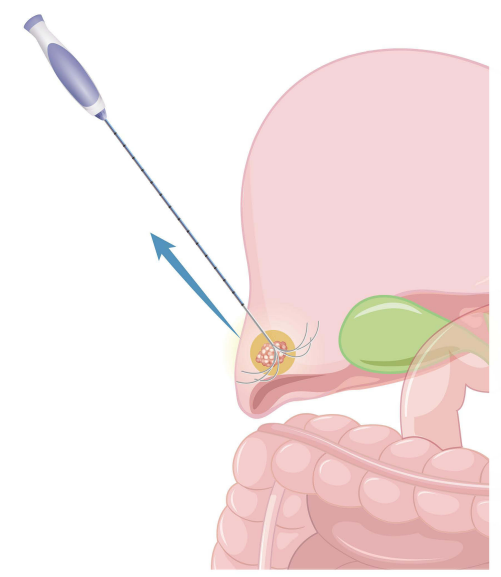

C

D

E

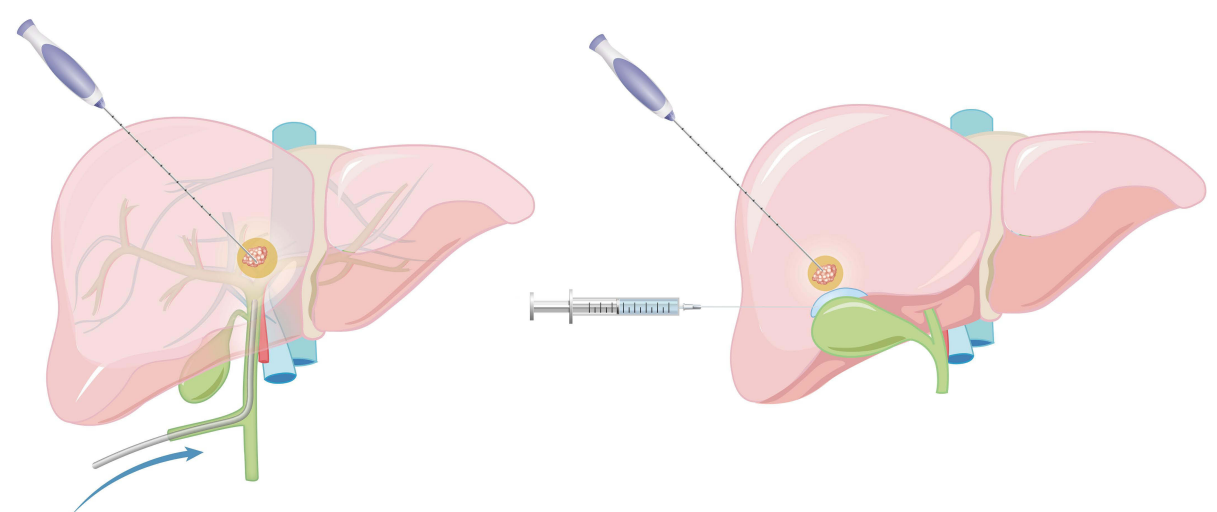

Figure 3 (A) Image-guided puncture needle is placed between liver sub-capsule and peritoneum, and water isolation protection is formed by injecting normal saline after placing drainage tube. (B and C) By lifting or prying up the tail of the applicator, target tumors can be dragged away from other organs around the liver, thereby further reducing the thermal damage caused by ablation. (D) Intermittently injecting glucose solution with a temperature around $4^{\circ} \mathrm{C}$ into the drainage tube can effectively protect the bile duct from thermal damage caused by RFA. (E) Injecting saline directly into the gallbladder bed and performing RFA after separating the gallbladder and liver capsule will also reduce the risk of thermal damage to the gallbladder wall.

coagulation, shorten the ablation time, and adapt to different tumor shapes and locations, so as to maximize the complete ablation rate. However, there is still no unified endpoint index or calculation method to evaluate the above- mentioned electrodes. In fact, clinicians do not care about the choice of electrodes. The key competitiveness of each type of radiofrequency electrode is the repeatability and accuracy of the ablation zone. 


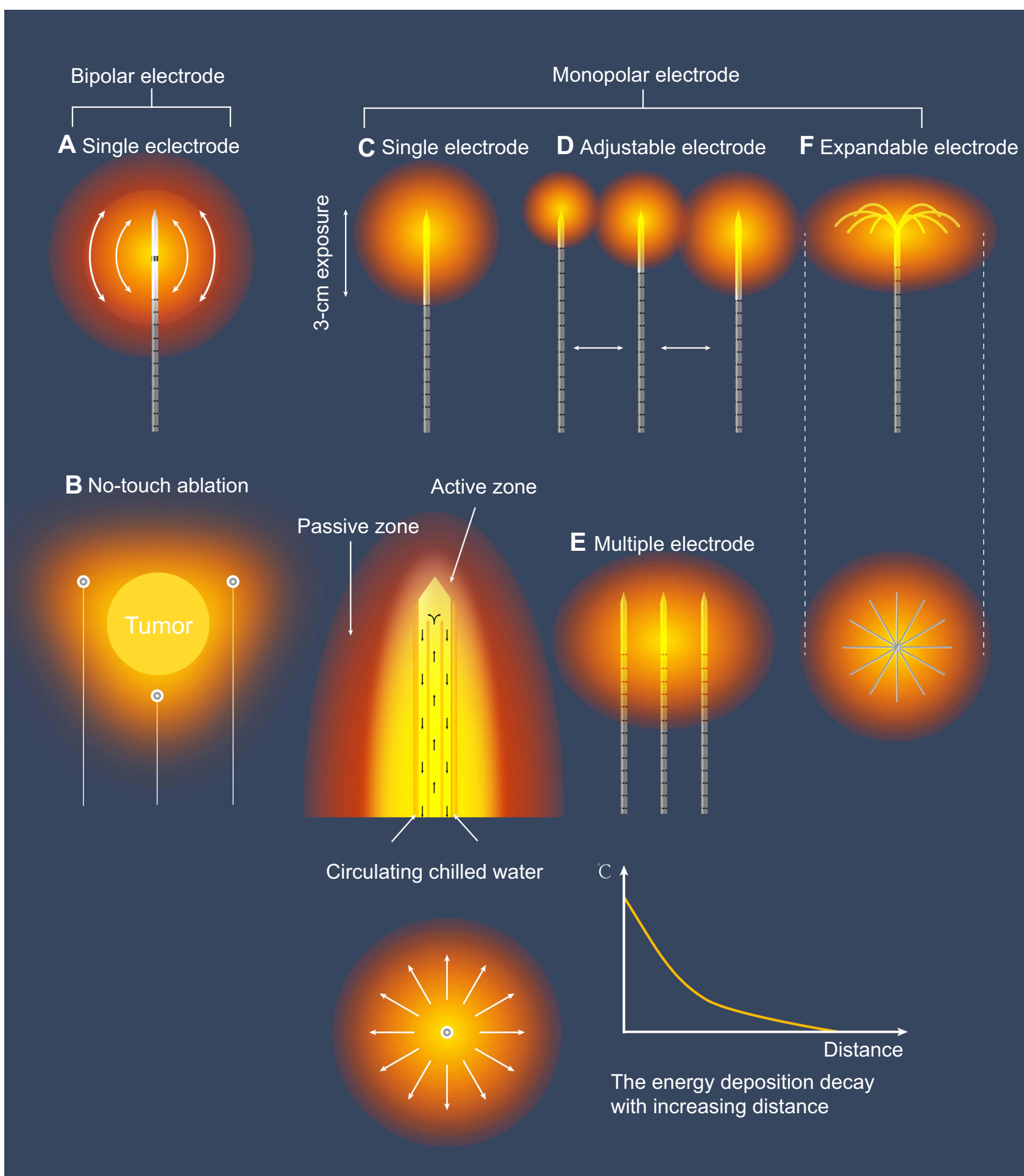

Figure 4 Various modalities and devices for radiofrequency ablation. (a) Bipolar electrode with no need of grounding pad. (b) Multi-bipolar system for no-touch ablation by placing the electrodes to surround but not directly puncture the target tumor. (c) Single electrode with internal cooling. (d) Adjustable electrode with variable ablative zone with one electrode. (e) Multiple monopolar electrodes with switch controller to enable larger ablative zone. (f) Expandable electrode with four to nine smaller electrodes inside.

\section{Microwave Ablation}

A new generation of microwave ablation equipment that combines internally cooled antenna, high-power transmission, and multiple antennas is widely used worldwide. ${ }^{77-85}$
The application of microwave (MWV) technology enables tissues to reach a higher temperature in a shorter period of time to obtain larger ablation zones. ${ }^{86}$ This breaks the limitation of small ablation zone of the RFA technology 


\begin{tabular}{|c|c|c|c|c|c|c|c|c|c|c|c|c|}
\hline 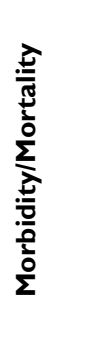 & 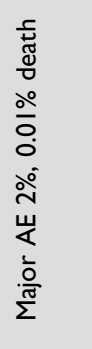 & 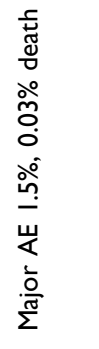 & 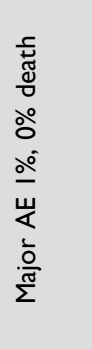 & 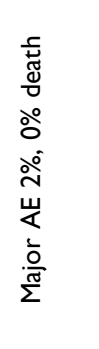 & 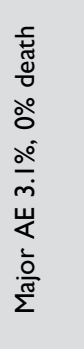 & 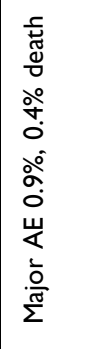 & 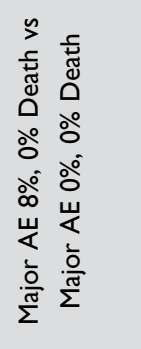 & 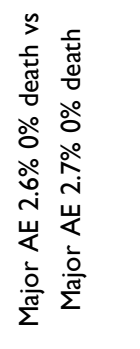 & 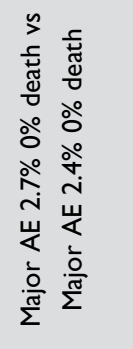 & 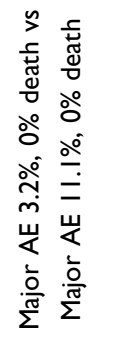 & 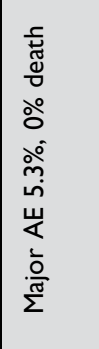 & 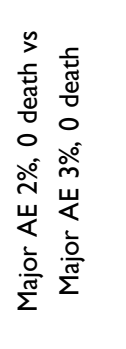 \\
\hline 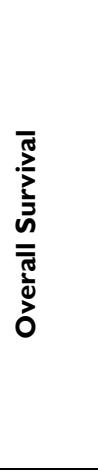 & 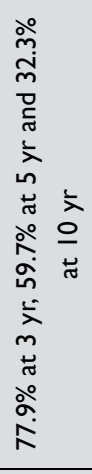 & 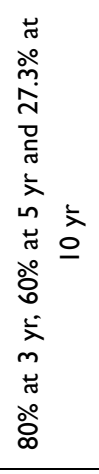 & 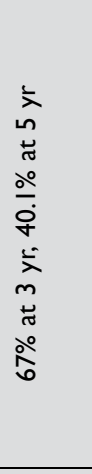 & 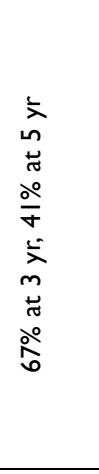 & 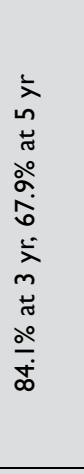 & 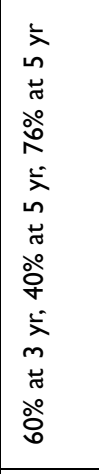 & 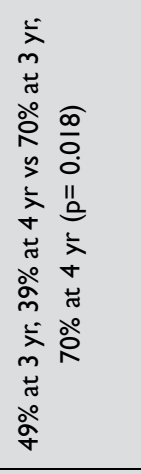 & 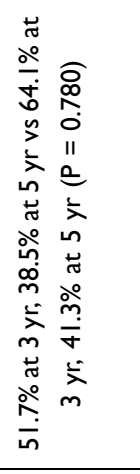 & 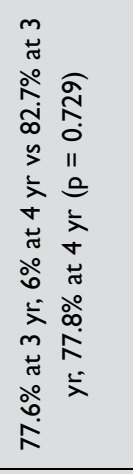 & 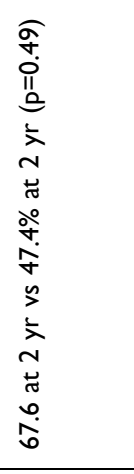 & 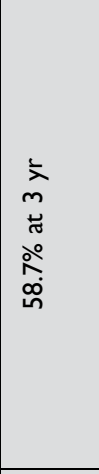 & 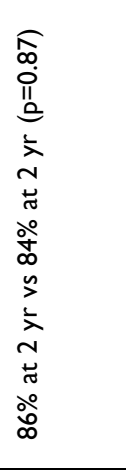 \\
\hline 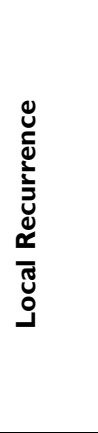 & 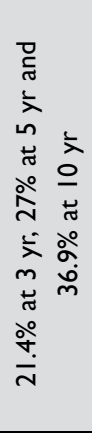 & 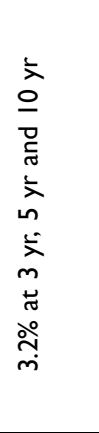 & 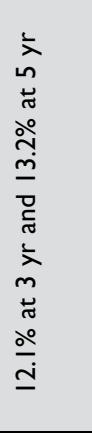 & 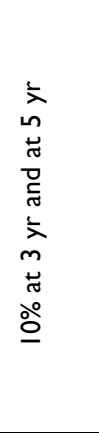 & 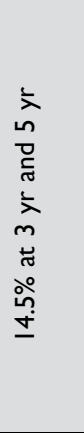 & 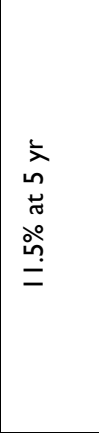 & 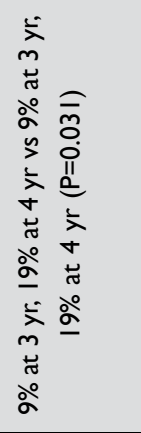 & 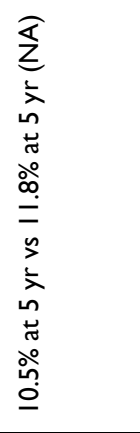 & 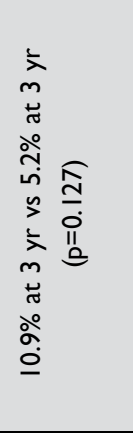 & 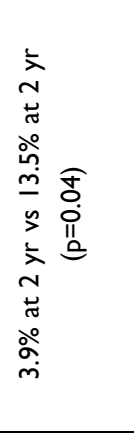 & 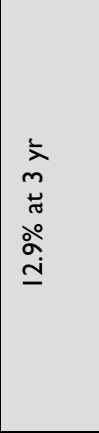 & 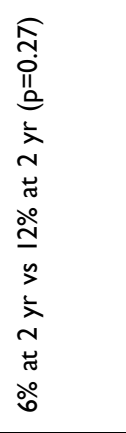 \\
\hline 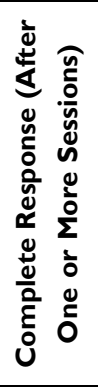 & $\begin{array}{l}\stackrel{\circ}{\circ} \\
\text { ळ }\end{array}$ & $\begin{array}{l}\stackrel{\circ}{\circ} \\
\text { مे }\end{array}$ & $\begin{array}{l}\stackrel{\circ}{\circ} \\
\text { ळ }\end{array}$ & ठั. & ஓ゚ & 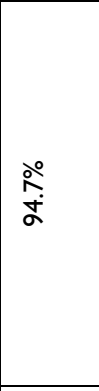 & $\S$ & 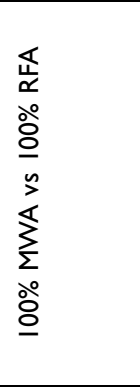 & 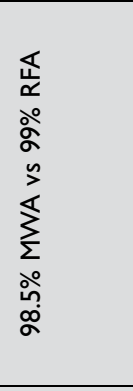 & 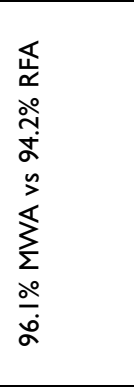 & $\begin{array}{l}\stackrel{\circ}{\alpha} \\
\dot{\alpha}\end{array}$ & 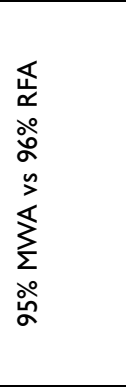 \\
\hline 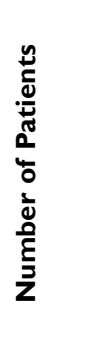 & 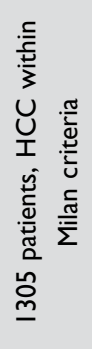 & 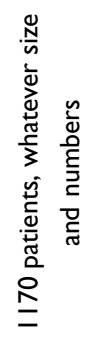 & 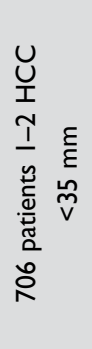 & 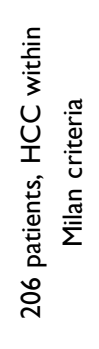 & 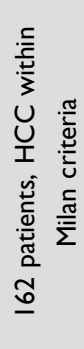 & 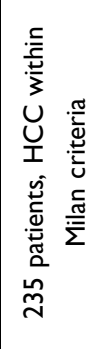 & 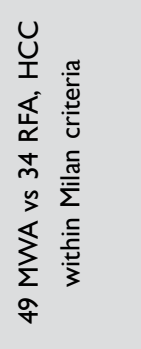 & 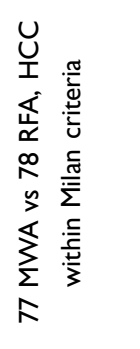 & 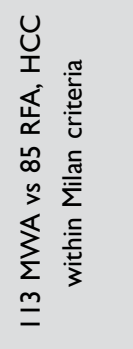 & 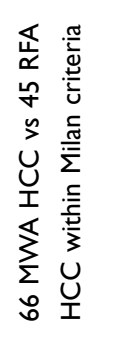 & 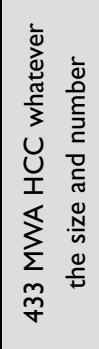 & 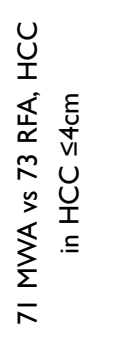 \\
\hline 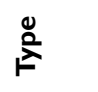 & 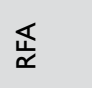 & $\underset{\underline{\alpha}}{\mathbb{4}}$ & $\underset{\frac{\pi}{\alpha}}{\mathbb{u}}$ & 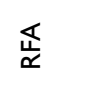 & $\underset{\mathbb{4}}{\mathbb{4}}$ & $\underset{\alpha}{\mathbb{\alpha}}$ & 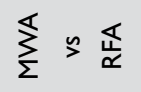 & $\sum_{\Sigma}^{\nwarrow} \sqrt{\frac{\pi}{\alpha}}$ & $\sum_{\Sigma}^{\mathbb{s}} \stackrel{\mathbb{4}}{\frac{\mathbb{x}}{\alpha}}$ & $\sum_{\Sigma}^{\overleftarrow{s}} s \frac{\mathbb{x}}{\alpha}$ & 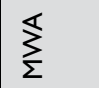 & 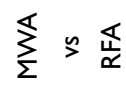 \\
\hline$\frac{0}{\frac{0}{\underline{V}}}$ & 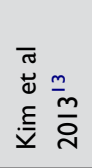 & 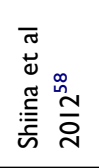 & 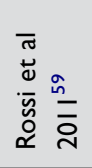 & 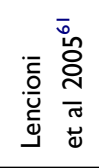 & 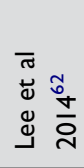 & 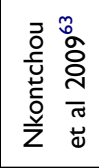 & 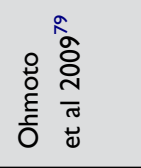 & 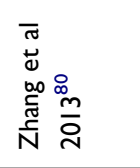 & 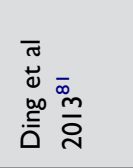 & 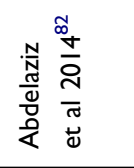 & $\begin{array}{l}\bar{\pi} \\
\tilde{0} \\
0 \\
\tilde{0} \\
\Sigma \\
\Sigma\end{array}$ & 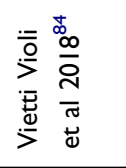 \\
\hline
\end{tabular}




\begin{tabular}{|c|c|c|c|c|c|c|c|c|c|c|c|}
\hline 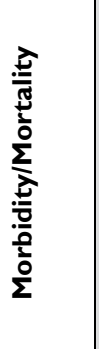 & 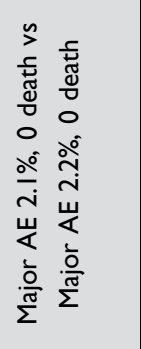 & 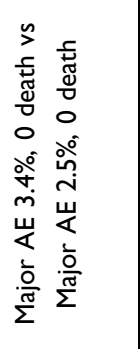 & 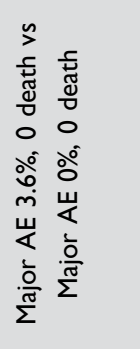 & 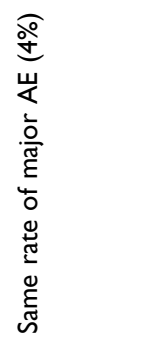 & 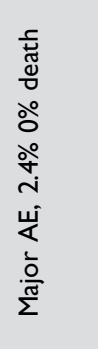 & 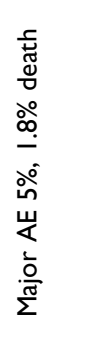 & 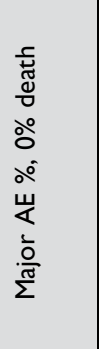 & 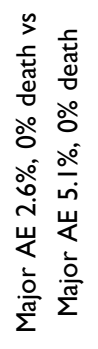 & 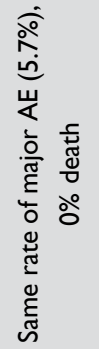 & 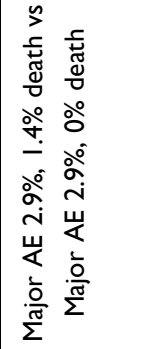 & 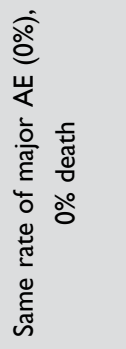 \\
\hline 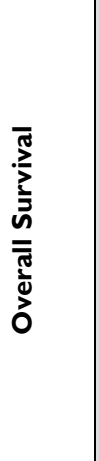 & 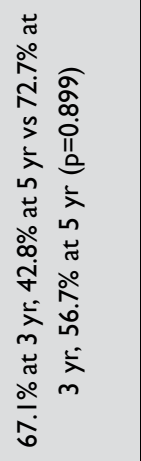 & 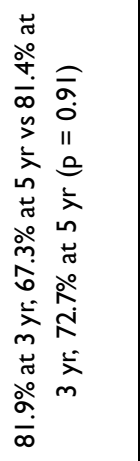 & 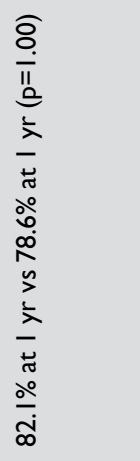 & 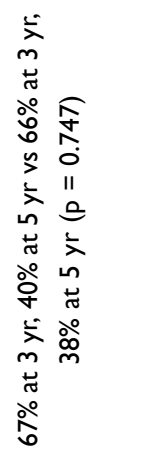 & 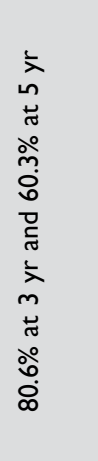 & 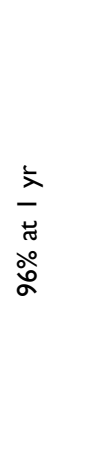 & $\S$ & 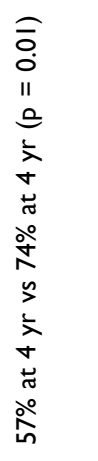 & 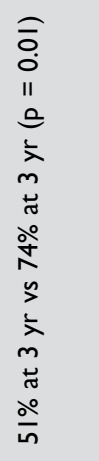 & 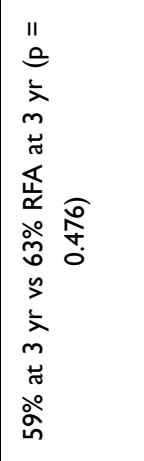 & 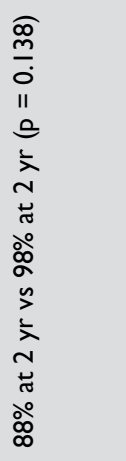 \\
\hline 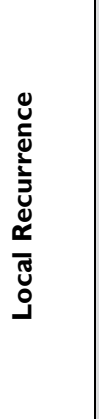 & $\S$ & 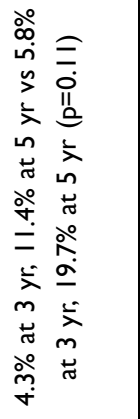 & 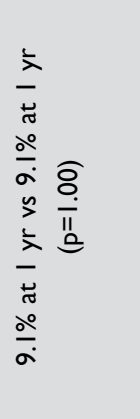 & 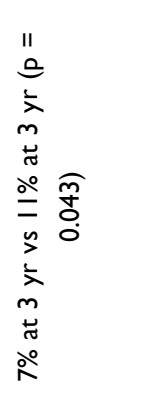 & 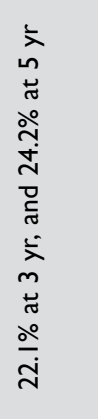 & 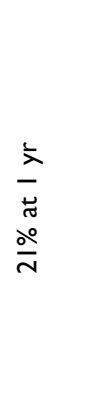 & 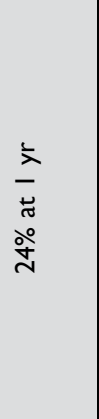 & 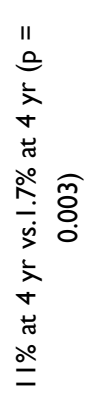 & 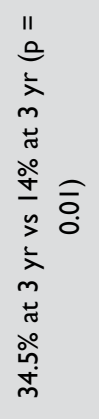 & 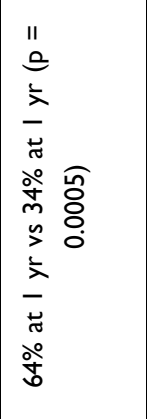 & 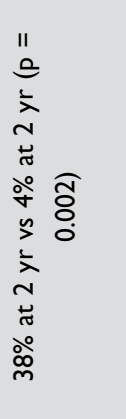 \\
\hline 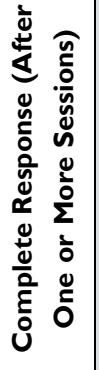 & 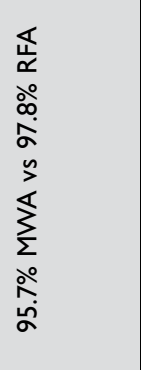 & 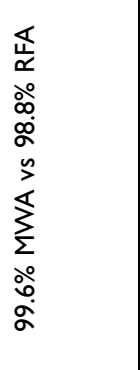 & $\S$ & 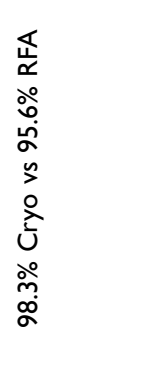 & $\frac{\circ}{\grave{\circ}}$ & ڤે & ळे & 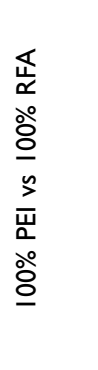 & 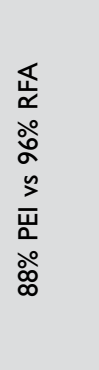 & 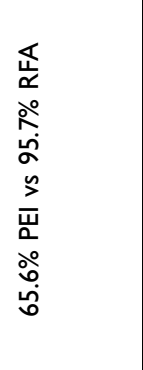 & 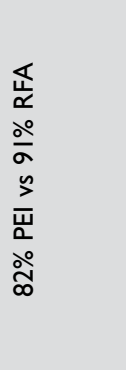 \\
\hline 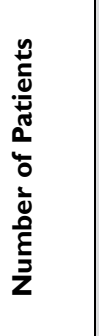 & 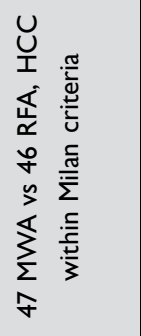 & 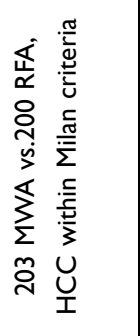 & 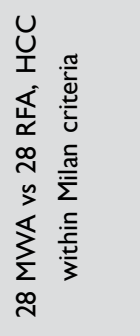 & 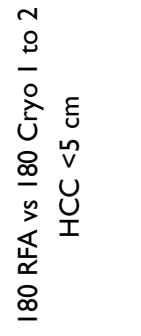 & 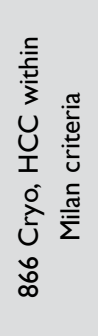 & 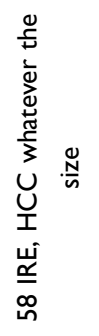 & 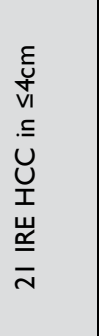 & 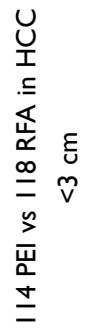 & 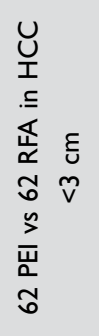 & 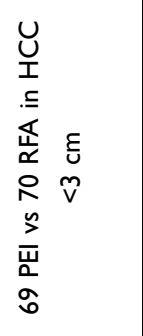 & 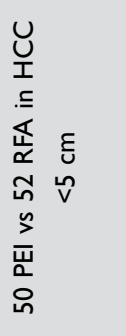 \\
\hline 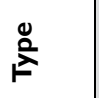 & 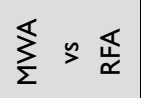 & 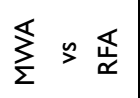 & 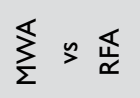 & 월 茫 & ํํํ & 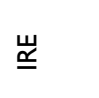 & 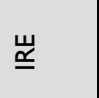 & 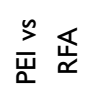 & 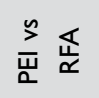 & 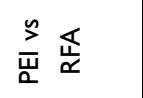 & 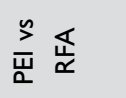 \\
\hline$\frac{\mathscr{0}}{\underline{\underline{L}}}$ & 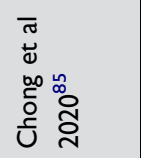 & 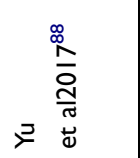 & 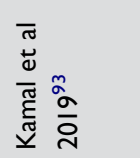 & 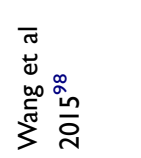 & 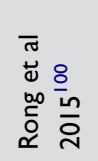 & 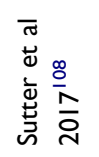 & 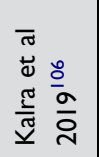 & 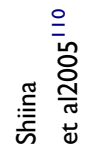 & 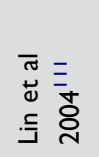 & 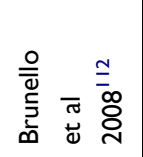 & 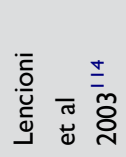 \\
\hline
\end{tabular}




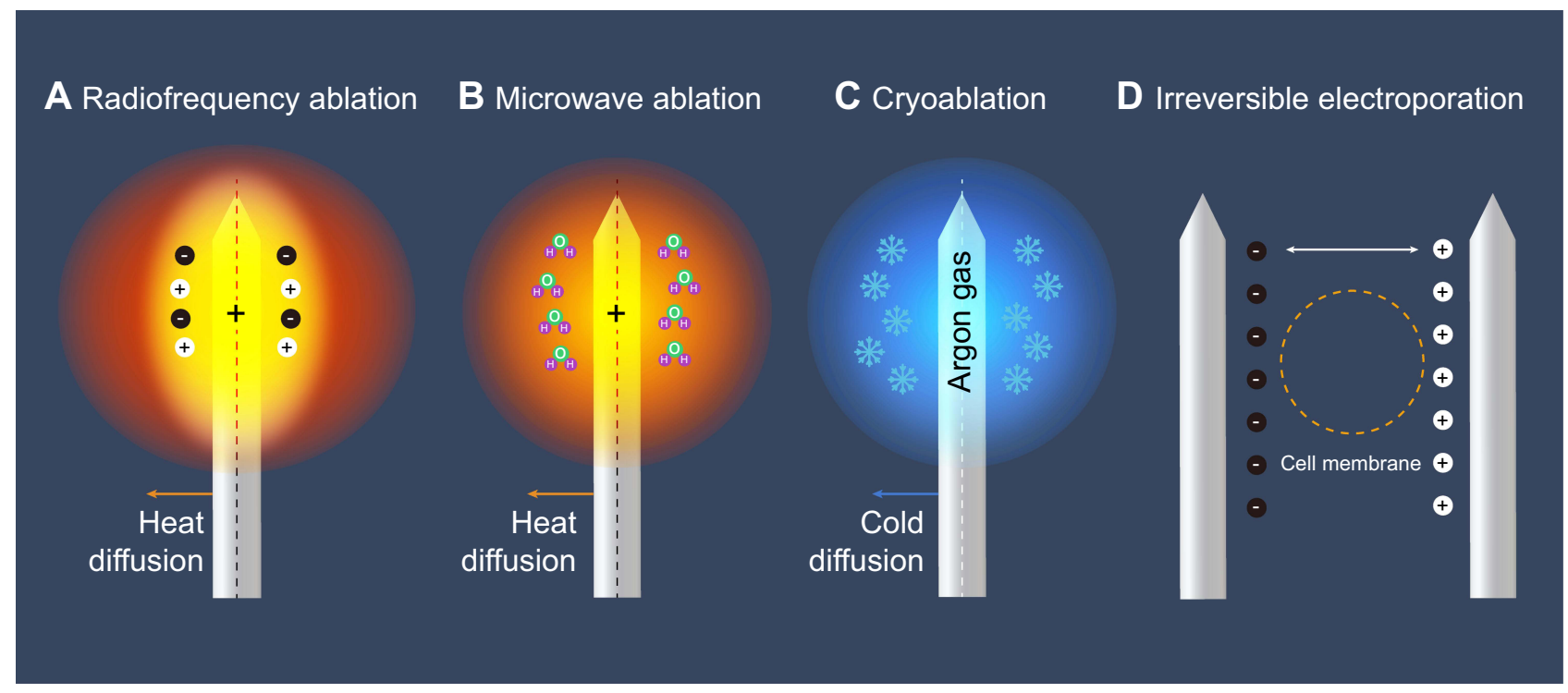

Figure 5 Various ablation technologies and principles. (A) Radiofrequency ablation. Application of oscillating electrical currents resulting in resistive heating surrounding an electrode and tissue hyperthermia. (B) Microwave ablation. Direct application of a propagating microwave energy level electromagnetic field to induce tissue hyperthermia via dielectric hysteresis. (C) Cryoablation. Changes in gas pressures result in cooling of a cryoprobe in direct thermal contact with tumor resulting in ice crystal formation and osmotic shock. (D) Irreversible electroporation. Alteration of transmembrane potentials to induce irreversible disruption of cell membrane integrity.

and reduces the potential risk of high tumor recurrence rate caused by the "heat sink effect" produced by adjacent large blood vessels. ${ }^{87}$ Compared with RFA, MWA is more promising because of its higher thermal efficiency. ${ }^{88}$ Currently, MWA has already been used to treat target tumors with a diameter of $\geq 5 \mathrm{~cm} .{ }^{89,90}$ However, in clinical practice, although MWV has the advantage of ablating a larger area of tissue in a shorter time, its local control effect for early-stage HCC is similar to that of RFA. ${ }^{27}$ A meta-analysis of 16 studies involving 2062 patients found that both MWA and RFA can be used as effective local treatments for early-stage HCC patients, because there is no significant difference between the two in terms of local tumor progression rate, overall survival rate, disease-free survival rate, and the incidence of adverse events. ${ }^{91}$ But it is obvious that MWA does have some advantages over RFA, such as faster ablation speed, shorter ablation time, less pain, less "heat sink effect", the ability to simultaneously treat multiple target tumors with multiple applicators, and the ability to treat target tumors with a diameter of 3-5 $\mathrm{cm}^{88,92-94}$ Therefore, when the diameter of a target tumor is $\geq 3 \mathrm{~cm}$ or the location of a target tumor is close to large blood vessels, MWA should be given priority. ${ }^{95}$ In terms of survival benefits, there is not enough evidence to prove that MWA has a better curative effect than RFA. The survival benefits of the two techniques warrant further comparative investigations.

\section{Cryoablation}

The major advantage of cryoablation is that when ultrasound or CT is used for image guidance, the contour of the "ice ball" can be clearly depicted to realize the visualization of ablation zone. ${ }^{96}$ However, the overall complication rate of cryoablation is as high as $50 \%,{ }^{97}$ and there are rare and serious complications compared with thermal ablation such as MWA and RFA. Such as cold shock, decreased platelet count, and bleeding. ${ }^{98}$ In addition, the mortality rate associated with cryoablation procedures is relatively high. ${ }^{99}$ Therefore, only a few medical centers use this ablation technique for $\mathrm{HCC}$ treatment. A previous study included 866 HCC patients who met the Milan criteria for liver transplantation. After cryoablation, $502(60 \%)$ of the patients were found to have tumor recurrence after a median follow-up of 31 months, and the 5-year survival rate was $60 \%{ }^{100}$ Nevertheless, because the "ice ball" can be used to conveniently monitor the ablation zone during the ablation process, cryoablation still possesses a great safety advantage for the treatment of tumors close to important ducts. However, it should be noted that the zone of coagulation does not exactly overlap the contours of the "ice ball"-its radius is about $4 \mathrm{~mm}$ shorter than that of the ice ball. ${ }^{101}$ Therefore, larger ablation zones must be created to ensure complete ablation of target tumors, but this usually requires more applicators. Studies describing clinical outcomes of cryoablation are 


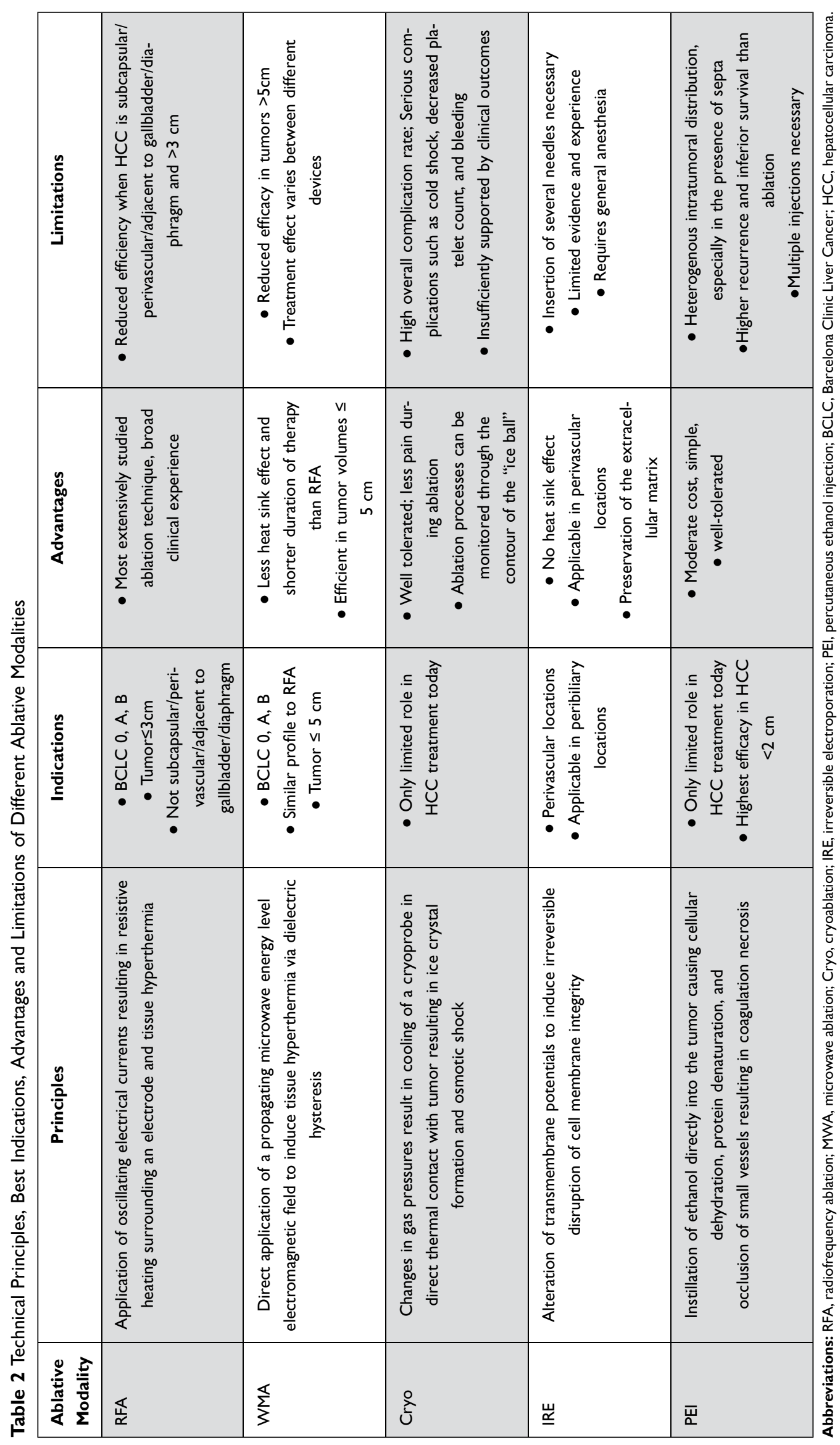


still limited, ${ }^{98,100,102}$ and the limitations of this technique seem to be similar to those of RFA and MWA. ${ }^{27,103}$

\section{Irreversible Electroporation}

Irreversible electroporation (IRE) is a nonthermal ablation technique. Since IRE can effectively protect the collagen skeleton, capillaries and bile ducts in non-tumor tissues, ${ }^{104}$ it is currently the best choice for the treatment of centrally located liver cancer with tumors adjacent to the main bile duct and portal vein. ${ }^{105,106}$ In addition, this technique seems to be more suitable than the thermal ablation techniques for patients with poor liver function and severe complications. ${ }^{107,108}$ Compared with thermal ablation, IRE is not restricted by the "heat sink effect" of surrounding blood flow. However, this therapy usually requires general anesthesia and its application is limited by patient conditions. For example, patients with sinus rhythm need to use blockers to avoid arrhythmia; patients with pacemakers are prohibited from using IRE; while patients treated with biliary-enteric anastomosis must use IRE with caution, because the risk of abscesses is significantly increased after IRE ablation in these patients. ${ }^{17}$ It should be noted that in order to achieve a predictable ablation zone, the applicators need to be placed as parallel as possible. It is recommended to use at least three applicators to ensure accurate positioning of the target tumor. ${ }^{109}$ Before IRE can be used with confidence like RFA or MWA, larger studies with longer follow-up times are still needed to evaluate the long-term efficacy of this ablative modality.

\section{Percutaneous Ethanol Injection}

Percutaneous ethanol injection (PEI) is suitable for HCC patients who have small tumor sizes and are not suitable for surgical resection due to poor liver functional reserve. However, many prospective randomized controlled trials (RCTs) and meta-analyses have shown that thermal ablation techniques are better than PEI. ${ }^{110-114}$ In clinical practice, thermal ablation techniques (especially RFA and MWA) are preferred, while PEI is usually not recommended. This is mainly because PEI can cause more discomfort to patients, and the required number of operations is more than that of RFA. In addition, air bubbles are easily formed during the injection of absolute ethanol, which will interfere with ultrasound guidance, making it difficult to observe the boundaries of target tumors. But for HCC with a tumor diameter of $\leq 2 \mathrm{~cm}$, PEI and RFA have similar therapeutic effects, and PEI has the advantages of lower cost and less equipment requirements. ${ }^{113}$ Therefore, PEI is still used to treat earlystage HCC patients who are not suitable for surgery and have small tumors. In addition, because the efficacy of PEI will not be affected by the "heat sink effect" and the safety of this technology is relatively high, PEI can be combined with thermal ablation to treat tumors adjacent to large blood vessels. ${ }^{6}$ Studies have shown that $90-100 \%$ of HCC patients with a tumor diameter of $\leq 2 \mathrm{~cm}$ completely responded to PEI; $70-80 \%$ of HCC patients with a tumor diameter of $\leq 3 \mathrm{~cm}$ completely responded to the treatment; while in HCC patients with larger tumors, the complete response rates to PEI were only $50-60 \% .{ }^{115-117}$ It can be seen that the complete response rates of HCC patients to PEI are closely related to the size of tumor. The main reason for the poor efficacy of PEI on large tumors is the existence of intratumoral compartment, which limits the spread of absolute ethanol. Therefore, in order to ensure the therapeutic effect, it is necessary to clarify the indications of PEI more strictly.

\section{Image Guidance Techniques}

An ideal image guidance technology should have the advantages of high sensitivity, real-time monitoring, easy operation, and low cost. Before treatment, image guidance techniques can provide physicians with information about the target tumor, such as its size, shape, infiltration range, anatomical relationship with adjacent organs, and local blood supply characteristics, based on which doctors can make treatment plans. During treatment, image guidance techniques can not only guide applicators into the target tumor along the preset path conveniently and accurately, but also clearly show the positional relationship between applicators and the surrounding tissues and changes in coagulated tissues, so as to monitor the entire ablation process in real time. After treatment, image guidance techniques can timely evaluate whether the expected curative effects of ablation are achieved and sensitively detect the occurrence of complications. At present, ultrasound is the most commonly used imaging technique for guiding percutaneous ablation of liver tumors, because it has the following advantages compared with other imaging techniques: real-time guidance on any imaging plane, no radiation, relatively simple operation, low cost, and can be used in outpatient departments. ${ }^{118}$

With high-HCC-risk populations (such as patients with chronic hepatitis B or liver cirrhosis) undergoing ultrasound screening every 6 months, ${ }^{119}$ as well as technological advancement in enhanced CT and enhanced MRI, more and more early-stage $\mathrm{HCC}$ cases with small tumor sizes 
(diameter $\leq 2 \mathrm{~cm}$ ) have been detected. ${ }^{120}$ Two studies on RFA have shown that in more than $90 \%$ of patients, target tumors with a diameter of $\leq 2 \mathrm{~cm}$ could be completely ablated, and the local recurrence rate was less than $1 \%{ }^{121,122}$ Therefore, early-stage HCC tumors within this size range are extremely suitable for percutaneous ablation. However, small HCC tumors may not be detected by ordinary ultrasound, especially in patients with a background of severe cirrhosis. ${ }^{123}$ In addition, there are areas in the liver that cannot be imaged by ultrasound, such as the top of the liver, the left lateral segment, and the area under the ribs. Ultrasound imaging is also susceptible to interference from abdominal gas and digestive tract. ${ }^{124}$ In order to better visualize target tumors, assistive techniques, such as artificial ascites and artificial hydrothorax, can be adopted. ${ }^{41,42}$ Contrast-enhanced ultrasound (CEUS) can also be applied to help identify lesions. ${ }^{125-128}$

Improving the visibility of target tumor in image guidance techniques is the key to improving the effect of tumor ablation, ${ }^{129}$ because a higher complete ablation rate is associated with a better prognosis. ${ }^{130,131}$ Therefore, it is necessary to optimize image guidance techniques and make full use of image information to guide treatment. At the same time, we need to have some basic understanding of the advantages, disadvantages and limitations of different imaging guidance techniques in clinical practice (Table 3). For ultrasound-guided RFA, needle electrodes are usually the first choice, because the tip of electrode can be clearly displayed under ultrasound, and the process of inserting electrodes into target tumors is similar to that of ultrasound-guided biopsy. ${ }^{132}$ On the contrary, expandable electrodes are more suitable for CT-guided ablations, because the tip of electrode can be more accurately placed on target tumors under the guidance of CT. However, CT cannot provide real-time guidance and has low contrast when visualizing HCC tumors. In addition, this method exposes both the patient and the operator to high doses of radiation, especially when multiple and overlapping ablations are required. ${ }^{133,134}$ There are some studies using MRI as the image guidance technique for percutaneous ablation. MRI has many advantages, such as near-real-time fluorescence imaging, higher sensitivity when detecting small target tumors, free choice of imaging plane, monitoring of thermal effects, and no ionizing radiation. However, due to insufficient MRI-compatible ablation equipment, complicated operation, prone to artifacts, and high cost, only a few medical centers specializing in MRI interventional treatment are performing MRI-guided ablations. ${ }^{135-137}$

Improving the visibility of target tumors before ablation and enhancing the sensitivity in detecting residual tumorous tissue after ablation are challenges for the current image guidance techniques. Nevertheless, achieving complete ablation of target tumors has always been our main goal. In recent years, fusion imaging has gradually been introduced into HCC percutaneous ablation therapy. ${ }^{124,129,138}$ Fusion imaging combines the advantages of different imaging methods, breaking the inherent limitations of each individual image guidance technique. It can detect $\mathrm{HCC}$ with a small tumor size (diameter $\leq 2 \mathrm{~cm}$ ) with higher sensitivity and specificity and can guide ablation therapy more accurately. ${ }^{139-141}$ A study indicated that in less than two-thirds of patients with recurrent subcentimeter-sized $(\leq 1 \mathrm{~cm})$ HCC, RFA guided by MRIconventional ultrasound fusion imaging showed $98.4 \%$ technical effectiveness. ${ }^{129}$ Conventional ultrasound can also be fused with Gd-EOB-DTPA-enhanced MRI to better display landmark structures in the liver, such as bile ducts and blood vessels. ${ }^{142}$ Even if the target tumor is still completely invisible, fusion imaging can also guide ablation based on the anatomical landmarks around the tumor and the virtual needle trajectory. In addition, a fusion imaging system incorporating a magnetic field generator and a sonographic transducer into an ultrasound probe was able to obtain more spatial information. ${ }^{143}$ However, because the morphology of the liver will be affected by the breathing motion of patient, small tumors and subcapsular tumors may still be difficult to accurately locate. ${ }^{144-146}$ An RCT involving 216 patients with a total of 243 tumors ( $\leq 5 \mathrm{~cm}$ in diameter) showed that real-time fusion imaging-guided RFA had similar technical effectiveness on tumors visible and invisible under conventional ultrasound $(96.1 \%$ versus $97.6 \%, P=0.295)$, and the cumulative incidence of local tumor progression in the 24-month follow-up was estimated to be $4.7 \%{ }^{138}$

Fusion imaging is also an effective method to assess ablative margin during surgery and during postoperative follow-up. Intraoperative assessment of ablative margin is essential to reduce local tumor recurrence and the possibility of a secondary ablation. ${ }^{147,148}$ Studies have shown that an insufficient ablation zone may be the reason for the higher local recurrence rate of percutaneous ablation compared with surgical resection. A sufficient ablation zone should include satellite nodules that cannot be detected by imaging methods. Satellite nodules are not common in 


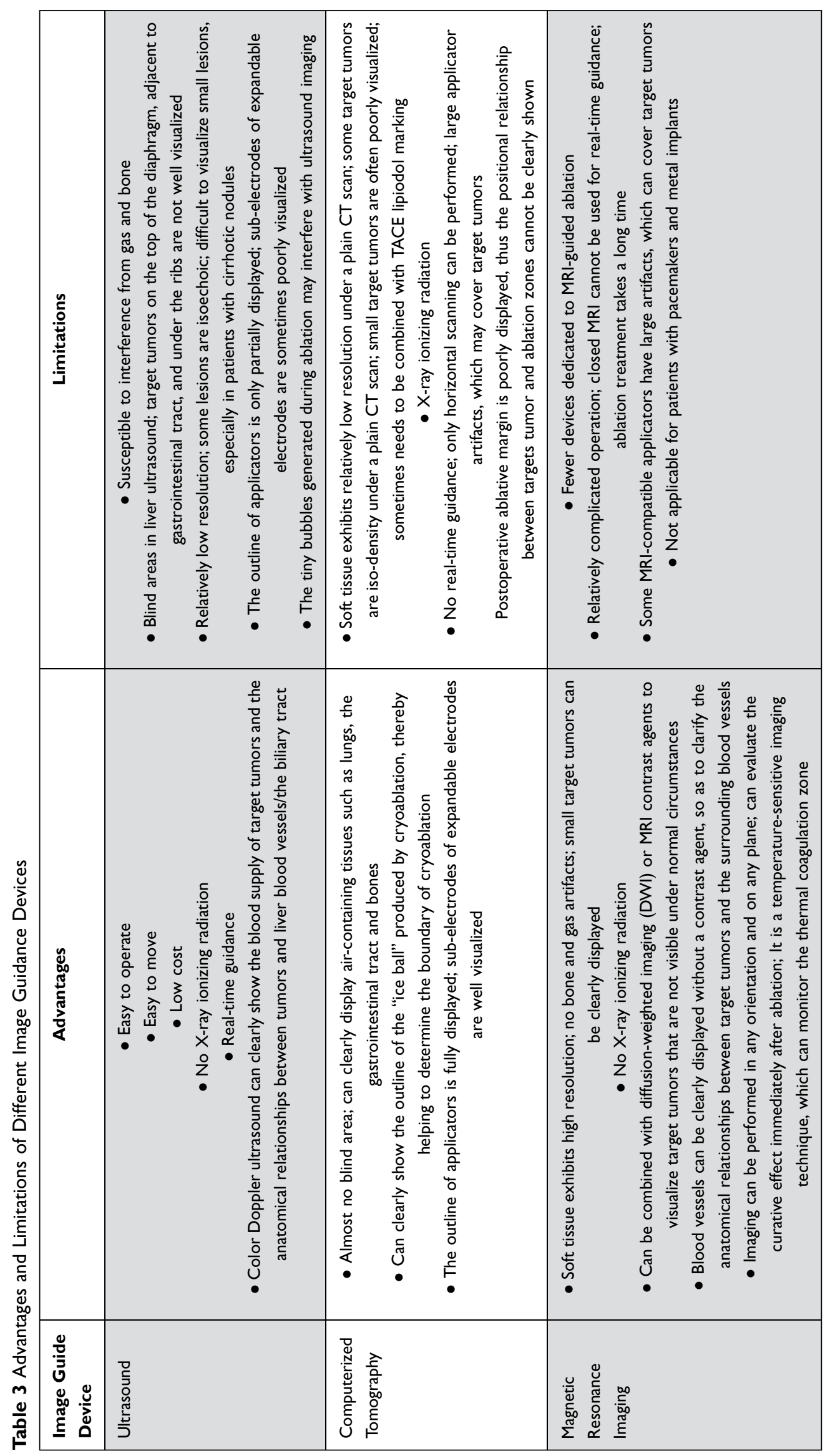


very-early-stage HCC patients, and the size of ablation zone may not have a large impact on the local tumor recurrence rate as in $\mathrm{HCC}$ patients with large tumors. ${ }^{149-151}$ However, it is still advocated that the ablation zone should include a 0.5 -to- $1-\mathrm{cm}$ "safety margin" all around the tumor margin to reduce the risk of local tumor progression caused by microvascular invasion and satellite nodules. ${ }^{152}$ It is worth noting that there are currently no high-quality studies using fusion imaging to assess the ablation zone after treatment.

\section{Combination Therapies}

The combination therapy of thermal ablation (such as RFA and MWA) and TACE can be used to optimize the curative effects and achieve a larger area of tumor destruction. TACE can improve the ablation efficiency by reducing the "heat sink effect" or increasing the sensitivity of tumor cells to high temperature. ${ }^{153}$ In addition, iodized oil can be used to mark satellite nodules that may be more commonly observed around large HCC tumors; the target ablation zone can also be defined based on the highdensity accumulation area of iodized oil in $\mathrm{HCC}^{154}$ so as to achieve more thorough and precise complete ablation. Several RCTs and retrospective studies have shown that, compared with RFA alone, the combination of RFA and TACE can achieve better survival benefits and lower local tumor progression rates when treating HCC tumors with a diameter of $3-5 \mathrm{~cm}^{155-158}$ The combination therapy could also achieve complete ablation of HCC tumors with a diameter of $>5 \mathrm{~cm} .{ }^{159-161}$ However, the implementation order and timing of TACE and RFA is a controversial issue - each proposal has certain theoretical advantages. Performing TACE first can reduce tumor blood perfusion to minimize the "heat sink effect"; while if RFA is performed first, TACE can be used to embolize incompletely ablated tumorous tissues at the ablative margin. Current evidence shows that the treatment regimen of TACE followed by ablation is superior to either RFA or TACE alone, and a larger coagulated area can be obtained. $^{162}$

\section{Conclusions}

Adopting $\mathrm{HCC}$ treatment regimens outside the scope of the guidelines will inevitably lead to lower survival benefits. Therefore, the existing ablative modalities are still facing many challenges: 1 . It is necessary to identify suitable patients who can switch from "palliative treatments" to "curative treatments". In reality, the formulation of treatment decisions involves a variety of factors, which will never be completely covered by a single practice guideline or a single number in the guideline. Therefore, a treatment regimen must be formulated by a multidisciplinary oncology discussion group composed of experienced hepatobiliary surgeons, hepatologists, interventional radiologists, oncologists and pathologists based on each patient's conditions. 2. The existing combinations of percutaneous ablation techniques need to be optimized, which requires us to make proper use of assistive technologies and correctly select the appropriate ablation equipment and image guidance methods. There is no recognized best combination of percutaneous ablation techniques. Therefore, further research is urgently needed to optimize the combinations of ablation techniques. 3. For a new technology/combination of technologies, we need to determine whether it can expand the indications for imageguided percutaneous ablation, improve operational safety, and improve the long-term prognosis of patients after percutaneous ablation. 4. The most critical factor in the successful implementation of ablation therapy is the operator. The operator needs to receive sufficient training, understand some rules of thumb, and be familiar with the working mechanisms and advantages and disadvantages of various ablation techniques. Capabilities such as accurate placement of applicators through image guidance are critical. However, it is equally important to thoroughly understand the expected imaging characteristics of successful and failed ablation procedures and the perioperative complications. Knowing and solving these aforementioned problems can better help us break the forbidden zone of percutaneous ablation.

\section{Funding}

The study was funded by Natural Science Foundation of Henan Province [162300410270]; Science and Technology Development Foundation of Henan Province [212102310115, 212102310135]; Henan Provincial Medical Science and Technology Project [SBGJ202002025, SBGJ202003009]; Henan Medical Science and Technology Innovation Talent Project [YXKC2020045].

\section{Disclosure}

All the authors declare that there is no conflict of interest. 


\section{References}

1. Pompili M, Saviano A, de Matthaeis N, et al. Long-term effectiveness of resection and radiofrequency ablation for single hepatocellular carcinoma $</=3 \mathrm{~cm}$. Results of a multicenter Italian survey. $J$ Hepatol. 2013;59(1):89-97. doi:10.1016/j. jhep.2013.03.009

2. Cho YK, Kim JK, Kim WT, Chung JW. Hepatic resection versus radiofrequency ablation for very early stage hepatocellular carcinoma: a Markov model analysis. Hepatology. 2010;51 (4):1284-1290. doi:10.1002/hep.23466

3. Kang TW, Kim JM, Rhim H, et al. Small hepatocellular carcinoma: radiofrequency ablation versus nonanatomic resection-propensity score analyses of long-term outcomes. Radiology. 2015;275(3):908-919. doi:10.1148/radiol.15141483

4. Peng ZW, Lin XJ, Zhang YJ, et al. Radiofrequency ablation versus hepatic resection for the treatment of hepatocellular carcinomas $2 \mathrm{~cm}$ or smaller: a retrospective comparative study. Radiology. 2012;262(3):1022-1033. doi:10.1148/radiol.11110817

5. Hung HH, Chiou YY, Hsia CY, et al. Survival rates are comparable after radiofrequency ablation or surgery in patients with small hepatocellular carcinomas. Clin Gastroenterol Hepatol. 2011;9(1):79-86. doi:10.1016/j.cgh.2010.08.018

6. Omata M, Cheng AL, Kokudo N, et al. Asia-Pacific clinical practice guidelines on the management of hepatocellular carcinoma: a 2017 update. Hepatol Int. 2017;11(4):317-370.

7. European Association for the Study of the Liver. Electronic address eee, European Association for the Study of the L. EASL Clinical Practice Guidelines: management of hepatocellular carcinoma. J Hepatol. 2018;69(1):182-236.

8. Zhou J, Sun HC, Wang Z, et al. Guidelines for diagnosis and treatment of primary liver cancer in China (2017 Edition). Liver Cancer. 2018;7(3):235-260. doi:10.1159/000488035

9. Korean Liver Cancer A, National Cancer Center GK. 2018 Korean Liver Cancer Association-National Cancer Center Korea Practice Guidelines for the Management of Hepatocellular Carcinoma. Korean $J$ Radiol. 2019;20(7):1042-1113. doi:10.3348/kjr.2019.0140

10. Heimbach JK, Kulik LM, Finn RS, et al. AASLD guidelines for the treatment of hepatocellular carcinoma. Hepatology. 2018;67 (1):358-380. doi:10.1002/hep.29086

11. Charriere B, Muscari F, Maulat C, et al. Outcomes of patients with hepatocellular carcinoma are determined in multidisciplinary team meetings. J Surg Oncol. 2017;115(3):330-336. doi:10.1002/ jso. 24500

12. Leoni S, Piscaglia F, Serio I, et al. Adherence to AASLD guidelines for the treatment of hepatocellular carcinoma in clinical practice: experience of the Bologna Liver Oncology Group. Dig Liver Dis. 2014;46(6):549-555. doi:10.1016/j.dld.2014.02.012

13. Kim YS, Lim HK, Rhim H, et al. Ten-year outcomes of percutaneous radiofrequency ablation as first-line therapy of early hepatocellular carcinoma: analysis of prognostic factors. $J$ Hepatol. 2013;58(1):89-97. doi:10.1016/j.jhep.2012.09.020

14. Solbiati L, Ierace T, Tonolini M, Cova L. Guidance and monitoring of radiofrequency liver tumor ablation with contrast-enhanced ultrasound. Eur J Radiol. 2004;51(Suppl):S19-23. doi:10.1016/j. ejrad.2004.03.035

15. Maeda M, Saeki I, Sakaida I, et al. Complications after radiofrequency ablation for hepatocellular carcinoma: a multicenter study involving 9411 Japanese patients. Liver Cancer. 2020;9 (1):50-62. doi:10.1159/000502744

16. Seror O. Ablative therapies: advantages and disadvantages of radiofrequency, cryotherapy, microwave and electroporation methods, or how to choose the right method for an individual patient? Diagn Interv Imaging. 2015;96(6):617-624. doi:10.1016/ j.diii.2015.04.007
17. Dai WC, Cheung TT, Chok KS, et al. Radiofrequency ablation versus transarterial chemoembolization for unresectable solitary hepatocellular carcinomas sized 5-8 cm. HPB (Oxford). 2015;17 (3):226-231. doi:10.1111/hpb.12324

18. Renzulli M, Tovoli F, Clemente A, et al. Ablation for hepatocellular carcinoma: beyond the standard indications. Med Oncol. 2020;37(4):23. doi:10.1007/s12032-020-01348-y

19. D'Avola D, Inarrairaegui M, Pardo F, et al. Prognosis of hepatocellular carcinoma in relation to treatment across BCLC stages. Ann Surg Oncol. 2011;18(7):1964-1971. doi:10.1245/s10434011-1551-4

20. Ho EY, Cozen ML, Shen H, et al. Expanded use of aggressive therapies improves survival in early and intermediate hepatocellular carcinoma. HPB (Oxford). 2014;16(8):758-767. doi:10.1111/hpb.12214

21. Pecorelli A, Lenzi B, Gramenzi A, et al. Curative therapies are superior to standard of care (transarterial chemoembolization) for intermediate stage hepatocellular carcinoma. Liver Int. 2017;37 (3):423-433. doi:10.1111/liv.13242

22. Nahon P, Vibert E, Nault JC, Ganne-Carrie N, Ziol M, Seror O. Optimizing curative management of hepatocellular carcinoma. Liver Int. 2020;40(Suppl 1):109-115. doi:10.1111/liv.14345

23. Padma S, Martinie JB, Iannitti DA. Liver tumor ablation: percutaneous and open approaches. $J$ Surg Oncol. 2009;100 (8):619-634. doi:10.1002/jso.21364

24. Callstrom MR, Charboneau JW. Technologies for ablation of hepatocellular carcinoma. Gastroenterology. 2008;134 (7):1831-1835. doi:10.1053/j.gastro.2008.05.022

25. Lencioni R, Cioni D, Della Pina C, Crocetti L. Hepatocellular carcinoma: new options for image-guided ablation. J Hepatobiliary Pancreat Sci. 2010;17(4):399-403. doi:10.1007/ s00534-009-0233-0

26. Lencioni R. Loco-regional treatment of hepatocellular carcinoma. Hepatology. 2010;52(2):762-773. doi:10.1002/hep.23725

27. Nault JC, Sutter O, Nahon P, Ganne-Carrie N, Seror O. Percutaneous treatment of hepatocellular carcinoma: state of the art and innovations. J Hepatol. 2018;68(4):783-797. doi:10.1016/ j.jhep.2017.10.004

28. Iezzi R, Pompili M, Posa A, Coppola G, Gasbarrini A, Bonomo L. Combined locoregional treatment of patients with hepatocellular carcinoma: state of the art. World J Gastroenterol. 2016;22(6):1935-1942. doi:10.3748/wjg.v22. i6.1935

29. Wang $\mathrm{Y}$, Zheng $\mathrm{J}$, Chen $\mathrm{H}$, et al. A prognostic nomogram for colorectal cancer liver metastases after percutaneous thermal ablation. Int J Hyperthermia. 2018;34(6):853-862. doi:10.1080/ 02656736.2017.1368095

30. Kloeckner R, Galle PR, Bruix J. Local and regional therapies for hepatocellular carcinoma. Hepatology. 2020. doi:10.1002/ hep. 31424

31. Giannini EG, Bucci L, Garuti F, et al. Patients with advanced hepatocellular carcinoma need a personalized management: a lesson from clinical practice. Hepatology. 2018;67 (5):1784-1796. doi:10.1002/hep.29668

32. Vitale A, Peck-Radosavljevic M, Giannini EG, et al. Personalized treatment of patients with very early hepatocellular carcinoma. J Hepatol. 2017;66(2):412-423. doi:10.1016/j.jhep.2016.09.012

33. Han K, Kim JH. Transarterial chemoembolization in hepatocellular carcinoma treatment: Barcelona clinic liver cancer staging system. World J Gastroenterol. 2015;21(36):10327-10335. doi:10.3748/wjg.v21.i36.10327

34. Kang TW, Lim HK, Lee MW, et al. Long-term therapeutic outcomes of radiofrequency ablation for subcapsular versus nonsubcapsular hepatocellular carcinoma: a Propensity Score Matched Study. Radiology. 2016;280(1):300-312. doi:10.1148/ radiol.2016151243 
35. Yuan Z, Xing A, Zheng J, Li W. Safety and technical feasibility of percutaneous ablation for lymph node metastases of hepatocellular carcinoma. Int $J$ Hyperthermia. 2019;36(1):160-168. doi:10.1080/02656736.2018.1542510

36. Llovet JM, Vilana R, Bru C, et al. Increased risk of tumor seeding after percutaneous radiofrequency ablation for single hepatocellular carcinoma. Hepatology. 2001;33(5):1124-1129. doi:10.1053/ jhep.2001.24233

37. Head HW, Dodd GD 3rd, Dalrymple NC, et al. Percutaneous radiofrequency ablation of hepatic tumors against the diaphragm: frequency of diaphragmatic injury. Radiology. 2007;243 (3):877-884. doi:10.1148/radiol.2433060157

38. Teratani T, Yoshida H, Shiina S, et al. Radiofrequency ablation for hepatocellular carcinoma in so-called high-risk locations. Hepatology. 2006;43(5):1101-1108. doi:10.1002/hep.21164

39. Nakagomi R, Tateishi R, Shiina S, et al. Drastically reduced neoplastic seeding related to radiofrequency ablation for hepatocellular carcinoma. Am J Gastroenterol. 2014;109(5):774-776. doi:10.1038/ajg.2014.42

40. Song I, Rhim H, Lim HK, Kim YS, Choi D. Percutaneous radiofrequency ablation of hepatocellular carcinoma abutting the diaphragm and gastrointestinal tracts with the use of artificial ascites: safety and technical efficacy in 143 patients. Eur Radiol. 2009;19 (11):2630-2640. doi:10.1007/s00330-009-1463-x

41. Rhim H, Lim HK, Kim YS, Choi D. Percutaneous radiofrequency ablation with artificial ascites for hepatocellular carcinoma in the hepatic dome: initial experience. AJR Am J Roentgenol. 2008;190 (1):91-98. doi:10.2214/AJR.07.2384

42. Koda M, Ueki M, Maeda Y, et al. Percutaneous sonographically guided radiofrequency ablation with artificial pleural effusion for hepatocellular carcinoma located under the diaphragm. AJR Am J Roentgenol. 2004;183(3):583-588. doi:10.2214/ajr.183.3.1830583

43. Wang CC, Kao JH. Artificial ascites is feasible and effective for difficult-to-ablate hepatocellular carcinoma. Hepatol Int. 2015;9 (4):514-519. doi:10.1007/s12072-015-9639-8

44. Kim JW, Shin SS, Heo SH, et al. Ultrasound-guided percutaneous radiofrequency ablation of liver tumors: how we do it safely and completely. Korean $J$ Radiol. 2015;16(6):1226-1239. doi:10.3348/kjr.2015.16.6.1226

45. Huang Q, Li J, Zeng Q, et al. Value of artificial ascites to assist thermal ablation of liver cancer adjacent to the gastrointestinal tract in patients with previous abdominal surgery. BMC Cancer. 2020;20(1):763. doi:10.1186/s12885-020-07261-x

46. Crocetti L, de Baere T, Lencioni R. Quality improvement guidelines for radiofrequency ablation of liver tumours. Cardiovasc Intervent Radiol. 2010;33(1):11-17. doi:10.1007/s00270-009-9736-y

47. Felker ER, Lee-Felker SA, Ajwichai K, et al. Intraductal cooling via a nasobiliary tube during radiofrequency ablation of central liver tumors reduces biliary injuries. AJR Am J Roentgenol. 2015;204(6):1329-1335. doi:10.2214/AJR.14.13788

48. Ohnishi T, Yasuda I, Nishigaki Y, et al. Intraductal chilled saline perfusion to prevent bile duct injury during percutaneous radiofrequency ablation for hepatocellular carcinoma. $J$ Gastroenterol Hepatol. 2008;23(8 Pt 2):e410-5. doi:10.1111/j.14401746.2007.05091.x

49. Levit E, Bruners P, Gunther RW, Mahnken AH. Bile aspiration and hydrodissection to prevent complications in hepatic RFA close to the gallbladder. Acta Radiol. 2012;53(9):1045-1048. doi:10.1258/ar.2012.120190

50. Garnon J, Koch G, Caudrelier J, et al. Hydrodissection of the gallbladder bed: a technique for ablations located close to the gallbladder. Cardiovasc Intervent Radiol. 2019;42(7):1029-1035. doi:10.1007/s00270-019-02218-5
51. Chang W, Lee JM, Lee SM, Han JK. No-touch radiofrequency ablation: a comparison of switching bipolar and switching monopolar ablation in ex vivo bovine liver. Korean J Radiol. 2017;18 (2):279-288. doi:10.3348/kjr.2017.18.2.279

52. N'Kontchou G, Nault JC, Sutter O, et al. Multibipolar radiofrequency ablation for the treatment of mass-forming and infiltrative hepatocellular carcinomas $>5 \mathrm{~cm}$ : long-term results. Liver Cancer. 2019;8(3):172-185. doi:10.1159/000489319

53. Seror O, N'Kontchou G, Nault JC, et al. Hepatocellular carcinoma within milan criteria: no-touch multibipolar radiofrequency ablation for treatment-long-term results. Radiology. 2016;280 (2):611-621. doi:10.1148/radiol.2016150743

54. Pua U. Extrahepatic biloma-potential imaging pitfall with "notouch" wedge ablation of subcapsular liver tumors. J Vasc Interv Radiol. 2014;25(4):655-656. doi:10.1016/j.jvir.2013.11.034

55. Lurje I, Czigany Z, Bednarsch J, et al. Treatment strategies for hepatocellular carcinoma (-) a multidisciplinary approach. Int J Mol Sci. 2019;20(6):1465. doi:10.3390/ijms20061465

56. Li D, Kang J, Golas BJ, Yeung VW, Madoff DC. Minimally invasive local therapies for liver cancer. Cancer Biol Med. 2014;11(4):217-236. doi:10.7497/j.issn.2095-3941.2014.04.001

57. Tateishi R, Fujiwara N. Precision locoregional therapies for hepatocellular carcinoma: percutaneous ablation and radiotherapy. In: Hoshida Y, editor. Hepatocellular Carcinoma: Translational Precision Medicine Approaches. Cham (CH); 2019:195-224. Springer Nature Switzerland AG.doi: 10.1007/978-3-030-21540810

58. Shiina S, Tateishi R, Arano T, et al. Radiofrequency ablation for hepatocellular carcinoma: 10-year outcome and prognostic factors. Am J Gastroenterol. 2012;107(4):569-77; quiz 78. doi:10.1038/ajg.2011.425

59. Rossi S, Ravetta V, Rosa L, et al. Repeated radiofrequency ablation for management of patients with cirrhosis with small hepatocellular carcinomas: a long-term cohort study. Hepatology. 2011;53(1):136-147. doi:10.1002/hep.23965

60. Cho YK, Kim JK, Kim MY, Rhim H, Han JK. Systematic review of randomized trials for hepatocellular carcinoma treated with percutaneous ablation therapies. Hepatology. 2009;49 (2):453-459. doi:10.1002/hep. 22648

61. Lencioni R, Cioni D, Crocetti L, et al. Early-stage hepatocellular carcinoma in patients with cirrhosis: long-term results of percutaneous image-guided radiofrequency ablation. Radiology. 2005;234(3):961-967. doi:10.1148/radiol.2343040350

62. Lee DH, Lee JM, Lee JY, et al. Radiofrequency ablation of hepatocellular carcinoma as first-line treatment: long-term results and prognostic factors in 162 patients with cirrhosis. Radiology. 2014;270(3):900-909. doi:10.1148/radiol.13130940

63. N'Kontchou G, Mahamoudi A, Aout M, et al. Radiofrequency ablation of hepatocellular carcinoma: long-term results and prognostic factors in 235 Western patients with cirrhosis. Hepatology. 2009;50(5):1475-1483. doi:10.1002/hep.23181

64. Yin XY, Xie XY, Lu MD, et al. Percutaneous thermal ablation of medium and large hepatocellular carcinoma: long-term outcome and prognostic factors. Cancer. 2009;115(9):1914-1923. doi: $10.1002 /$ cncr.24196

65. Lu DS, Raman SS, Vodopich DJ, Wang M, Sayre J, Lassman C. Effect of vessel size on creation of hepatic radiofrequency lesions in pigs: assessment of the "heat sink" effect. AJR Am $J$ Roentgenol. 2002;178(1):47-51. doi:10.2214/ajr.178.1.1780047

66. Rossi S, Garbagnati F, Lencioni R, et al. Percutaneous radio-frequency thermal ablation of nonresectable hepatocellular carcinoma after occlusion of tumor blood supply. Radiology. 2000;217(1):119-126. doi:10.1148/radiology.217.1. r00se02119 
67. Yamasaki T, Kimura T, Kurokawa F, et al. Percutaneous radiofrequency ablation with cooled electrodes combined with hepatic arterial balloon occlusion in hepatocellular carcinoma. J Gastroenterol. 2005;40(2):171-178. doi:10.1007/s00535-004-1516-5

68. Ahmed M, Brace CL, Lee FT Jr, Goldberg SN. Principles of and advances in percutaneous ablation. Radiology. 2011;258 (2):351-369. doi:10.1148/radiol.10081634

69. Solbiati L, Ierace T, Gennaro N, Muglia R, Cosman ER Jr, Goldberg SN. Percutaneous radiofrequency ablation of HCC: reduced ablation duration and increased ablation size using single, internally cooled electrodes with an optimized pulsing algorithm. Int $J$ Hyperthermia. 2020;37(1):861-867. doi:10.1080/ 02656736.2020.1790678

70. Woo S, Lee JM, Yoon JH, et al. Small- and medium-sized hepatocellular carcinomas: monopolar radiofrequency ablation with a multiple-electrode switching system-mid-term results. Radiology. 2013;268(2):589-600. doi:10.1148/radiol.13121736

71. McGahan JP, Loh S, Boschini FJ, et al. Maximizing parameters for tissue ablation by using an internally cooled electrode. Radiology. 2010;256(2):397-405. doi:10.1148/ radiol.09090662

72. Goldberg SN, Solbiati L, Hahn PF, et al. Large-volume tissue ablation with radio frequency by using a clustered, internally cooled electrode technique: laboratory and clinical experience in liver metastases. Radiology. 1998;209(2):371-379. doi:10.1148/ radiology.209.2.9807561

73. Goldberg SN, Gazelle GS, Solbiati L, Rittman WJ, Mueller PR. Radiofrequency tissue ablation: increased lesion diameter with a perfusion electrode. Acad Radiol. 1996;3(8):636-644. doi:10.1016/S1076-6332(96)80188-7

74. Lencioni R, Goletti O, Armillotta N, et al. Radio-frequency thermal ablation of liver metastases with a cooled-tip electrode needle: results of a pilot clinical trial. Eur Radiol. 1998;8 (7):1205-1211. doi:10.1007/s003300050536

75. Shibata T, Shibata T, Maetani Y, Isoda H, Hiraoka M. Radiofrequency ablation for small hepatocellular carcinoma: prospective comparison of internally cooled electrode and expandable electrode. Radiology. 2006;238(1):346-353. doi:10.1148/ radiol.2381041848

76. Cha J, Kim YS, Rhim H, Lim HK, Choi D, Lee MW. Radiofrequency ablation using a new type of internally cooled electrode with an adjustable active tip: an experimental study in ex vivo bovine and in vivo porcine livers. Eur J Radiol. 2011;77 (3):516-521. doi:10.1016/j.ejrad.2009.09.011

77. Cazzato RL, De Marini P, Leclerc L, et al. Large nearly spherical ablation zones are achieved with simultaneous multi-antenna microwave ablation applied to treat liver tumours. Eur Radiol. 2020;30(2):971-975. doi:10.1007/s00330-019-06431-1

78. Imajo K, Tomeno W, Kanezaki M, et al. New microwave ablation system for unresectable liver tumors that forms large, spherical ablation zones. J Gastroenterol Hepatol. 2018;33(12):2007-2014. doi:10.1111/jgh.14294

79. Ohmoto K, Yoshioka N, Tomiyama Y, et al. Comparison of therapeutic effects between radiofrequency ablation and percutaneous microwave coagulation therapy for small hepatocellular carcinomas. J Gastroenterol Hepatol. 2009;24(2):223-227. doi:10.1111/j.1440-1746.2008.05596.x

80. Zhang L, Wang N, Shen Q, Cheng W, Qian GJ. Therapeutic efficacy of percutaneous radiofrequency ablation versus microwave ablation for hepatocellular carcinoma. PLoS One. 2013;8 (10):e76119. doi:10.1371/journal.pone.0076119

81. Ding J, Jing X, Liu J, et al. Comparison of two different thermal techniques for the treatment of hepatocellular carcinoma. Eur J Radiol. 2013;82(9):1379-1384. doi:10.1016/ j.ejrad.2013.04.025
82. Abdelaziz A, Elbaz T, Shousha HI, et al. Efficacy and survival analysis of percutaneous radiofrequency versus microwave ablation for hepatocellular carcinoma: an Egyptian multidisciplinary clinic experience. Surg Endosc. 2014;28(12):3429-3434. doi:10.1007/s00464-014-3617-4

83. Ma S, Ding M, Li J, et al. Ultrasound-guided percutaneous microwave ablation for hepatocellular carcinoma: clinical outcomes and prognostic factors. J Cancer Res Clin Oncol. 2017;143(1):131-142. doi:10.1007/s00432-016-2266-5

84. Vietti Violi N, Duran R, Guiu B, et al. Efficacy of microwave ablation versus radiofrequency ablation for the treatment of hepatocellular carcinoma in patients with chronic liver disease: a randomised controlled Phase 2 trial. Lancet Gastroenterol Hepatol. 2018;3 (5):317-325. doi:10.1016/S2468-1253(18)30029-3

85. Chong CCN, Lee KF, Cheung SYS, et al. Prospective double-blinded randomized controlled trial of microwave versus radiofrequency ablation for hepatocellular carcinoma (McRFA trial). HPB (Oxford). 2020;22(8):1121-1127. doi:10.1016/j. hpb.2020.01.008

86. Hinshaw JL, Lubner MG, Ziemlewicz TJ, Lee FT Jr, Brace CL. Percutaneous tumor ablation tools: microwave, radiofrequency, or cryoablation-what should you use and why? Radiographics. 2014;34(5):1344-1362. doi:10.1148/rg.345140054

87. Huang S, Yu J, Liang P, et al. Percutaneous microwave ablation for hepatocellular carcinoma adjacent to large vessels: a long-term follow-up. Eur $J$ Radiol. 2014;83(3):552-558. doi:10.1016/j.ejrad.2013.12.015

88. Yu J, Yu XL, Han ZY, et al. Percutaneous cooled-probe microwave versus radiofrequency ablation in early-stage hepatocellular carcinoma: a Phase III randomised controlled trial. Gut. 2017;66 (6):1172-1173.

89. Zhang TQ, Huang ZM, Shen JX, et al. Safety and effectiveness of multi-antenna microwave ablation-oriented combined therapy for large hepatocellular carcinoma. Therap Adv Gastroenterol. 2019;12:1756284819862966. doi:10.1177/1756284819862966

90. Xu Y, Shen Q, Wang N, et al. Percutaneous microwave ablation of 5-6 cm unresectable hepatocellular carcinoma: local efficacy and long-term outcomes. Int $J$ Hyperthermia. 2017;33 (3):247-254. doi:10.1080/02656736.2016.1239842

91. Huo YR, Eslick GD. Microwave ablation compared to radiofrequency ablation for hepatic lesions: a meta-analysis. J Vasc Interv Radiol. 2015;26(8):1139-46 e2. doi:10.1016/j.jvir.2015.04.004

92. Qian GJ, Wang N, Shen Q, et al. Efficacy of microwave versus radiofrequency ablation for treatment of small hepatocellular carcinoma: experimental and clinical studies. Eur Radiol. 2012;22 (9):1983-1990. doi:10.1007/s00330-012-2442-1

93. Kamal A, Elmoety AAA, Rostom YAM, Shater MS, Lashen SA Percutaneous radiofrequency versus microwave ablation for management of hepatocellular carcinoma: a randomized controlled trial. J Gastrointest Oncol. 2019;10(3):562-571. doi:10.21037/ jgo.2019.01.34

94. Shiina S, Sato K, Tateishi R, et al. Percutaneous ablation for hepatocellular carcinoma: comparison of various ablation techniques and surgery. Can J Gastroenterol Hepatol. 2018;2018:4756147. doi:10.1155/2018/4756147

95. Izzo F, Granata V, Grassi R, et al. Radiofrequency ablation and microwave ablation in liver tumors: an update. Oncologist. 2019;24(10):e990-e1005. doi:10.1634/theoncologist.2018-0337

96. Morris DL, Horton MD, Dilley AV, Warlters A, Clingan PR. Treatment of hepatic metastases by cryotherapy and regional cytotoxic perfusion. Gut. 1993;34(9):1156-1157. doi:10.1136/ gut.34.9.1156

97. Seifert JK, Junginger T, Morris DL. A collective review of the world literature on hepatic cryotherapy. $J R$ Coll Surg Edinb. 1998;43(3):141-154. 
98. Wang C, Wang H, Yang W, et al. Multicenter randomized controlled trial of percutaneous cryoablation versus radiofrequency ablation in hepatocellular carcinoma. Hepatology. 2015;61 (5):1579-1590. doi:10.1002/hep.27548

99. Jansen MC, van Hillegersberg $R$, Chamuleau RA, van Delden OM, Gouma DJ, van Gulik TM. Outcome of regional and local ablative therapies for hepatocellular carcinoma: a collective review. Eur J Surg Oncol. 2005;31(4):331-347. doi:10.1016/j.ejso.2004.10.011

100. Rong G, Bai W, Dong Z, et al. Long-term outcomes of percutaneous cryoablation for patients with hepatocellular carcinoma within Milan criteria. PLoS One. 2015;10(4):e0123065. doi:10.1371/journal.pone. 0123065

101. Weber SM, Lee FT Jr, Warner TF, Chosy SG, Mahvi DM. Hepatic cryoablation: US monitoring of extent of necrosis in normal pig liver. Radiology. 1998;207(1):73-77. doi:10.1148/ radiology.207.1.9530301

102. Xu J, Noda C, Erickson A, et al. Radiofrequency ablation vs. cryoablation for localized hepatocellular carcinoma: a propensity-matched Population Study. Anticancer Res. 2018;38 (11):6381-6386. doi:10.21873/anticanres. 12997

103. Kim R, Kang TW, Cha DI, et al. Percutaneous cryoablation for perivascular hepatocellular carcinoma: therapeutic efficacy and vascular complications. Eur Radiol. 2019;29(2):654-662. doi:10.1007/s00330-018-5617-6

104. Cheng RG, Bhattacharya R, Yeh MM, Padia SA. Irreversible electroporation can effectively ablate hepatocellular carcinoma to complete pathologic necrosis. J Vasc Interv Radiol. 2015;26 (8):1184-1188. doi:10.1016/j.jvir.2015.05.014

105. Distelmaier M, Barabasch A, Heil P, et al. Midterm safety and efficacy of irreversible electroporation of malignant liver tumors located close to major portal or hepatic veins. Radiology. 2017;285(3):1023-1031. doi:10.1148/radiol.2017161561

106. Kalra N, Gupta P, Gorsi U, et al. Irreversible electroporation for unresectable hepatocellular carcinoma: initial experience. Cardiovasc Intervent Radiol. 2019;42(4):584-590. doi:10.1007/ s00270-019-02164-2

107. Bhutiani N, Philips P, Scoggins CR, McMasters KM, Potts MH, Martin RC. Evaluation of tolerability and efficacy of irreversible electroporation (IRE) in treatment of Child-Pugh B (7/8) hepatocellular carcinoma (HCC). HPB (Oxford). 2016;18(7):593-599. doi:10.1016/j.hpb.2016.03.609

108. Sutter O, Calvo J, N'Kontchou G, et al. Safety and efficacy of irreversible electroporation for the treatment of hepatocellular carcinoma not amenable to thermal ablation techniques: a retrospective single-center case series. Radiology. 2017;284 (3):877-886. doi:10.1148/radiol.2017161413

109. Silk M, Tahour D, Srimathveeravalli G, Solomon SB, Thornton RH. The state of irreversible electroporation in interventional oncology. Semin Intervent Radiol. 2014;31(2):111-117. doi:10.1055/s-0034-1373785

110. Shiina S, Teratani T, Obi S, et al. A randomized controlled trial of radiofrequency ablation with ethanol injection for small hepatocellular carcinoma. Gastroenterology. 2005;129(1):122-130. doi:10.1053/j.gastro.2005.04.009

111. Lin SM, Lin CJ, Lin CC, Hsu CW, Chen YC. Radiofrequency ablation improves prognosis compared with ethanol injection for hepatocellular carcinoma $<$ or $=4 \mathrm{~cm}$. Gastroenterology. 2004;127 (6):1714-1723. doi:10.1053/j.gastro.2004.09.003

112. Brunello F, Veltri A, Carucci P, et al. Radiofrequency ablation versus ethanol injection for early hepatocellular carcinoma: a randomized controlled trial. Scand J Gastroenterol. 2008;43 (6):727-735. doi:10.1080/00365520701885481
113. Germani G, Pleguezuelo M, Gurusamy K, Meyer T, Isgro G, Burroughs AK. Clinical outcomes of radiofrequency ablation, percutaneous alcohol and acetic acid injection for hepatocelullar carcinoma: a meta-analysis. J Hepatol. 2010;52(3):380-388. doi:10.1016/j.jhep.2009.12.004

114. Lencioni RA, Allgaier HP, Cioni D, et al. Small hepatocellular carcinoma in cirrhosis: randomized comparison of radio-frequency thermal ablation versus percutaneous ethanol injection. Radiology. 2003;228(1):235-240. doi:10.1148/ radiol.2281020718

115. El-Serag HB, Marrero JA, Rudolph L, Reddy KR. Diagnosis and treatment of hepatocellular carcinoma. Gastroenterology. 2008;134(6):1752-1763. doi:10.1053/j.gastro.2008.02.090

116. Lencioni R, Llovet JM. Percutaneous ethanol injection for hepatocellular carcinoma: alive or dead? J Hepatol. 2005;43 (3):377-380. doi:10.1016/j.jhep.2005.06.001

117. Livraghi T, Bolondi L, Lazzaroni S, et al. Percutaneous ethanol injection in the treatment of hepatocellular carcinoma in cirrhosis. A study on 207 patients. Cancer. 1992;69(4):925-929. doi:10.1002/1097-0142(19920215)69:4<925::AIDCNCR2820690415>3.0.CO;2-G

118. Rhim H, Lee MH, Kim YS, Choi D, Lee WJ, Lim HK. Planning sonography to assess the feasibility of percutaneous radiofrequency ablation of hepatocellular carcinomas. AJR Am J Roentgenol. 2008;190(5):1324-1330. doi:10.2214/AJR.07.2970

119. Bruix J, Sherman M; American Association for the Study of Liver D. Management of hepatocellular carcinoma: an update. Hepatology. 2011;53(3):1020-1022. doi:10.1002/hep.24199

120. Roskams T, Kojiro M. Pathology of early hepatocellular carcinoma: conventional and molecular diagnosis. Semin Liver Dis. 2010;30(1):17-25. doi:10.1055/s-0030-1247129

121. Livraghi T, Meloni F, Di Stasi M, et al. Sustained complete response and complications rates after radiofrequency ablation of very early hepatocellular carcinoma in cirrhosis: is resection still the treatment of choice? Hepatology. 2008;47(1):82-89. doi:10.1002/hep.21933

122. Cucchetti A, Piscaglia F, Cescon M, et al. Cost-effectiveness of hepatic resection versus percutaneous radiofrequency ablation for early hepatocellular carcinoma. J Hepatol. 2013;59(2):300-307. doi:10.1016/j.jhep.2013.04.009

123. Lee MW, Kim YJ, Park HS, et al. Targeted sonography for small hepatocellular carcinoma discovered by CT or MRI: factors affecting sonographic detection. AJR Am J Roentgenol. 2010;194(5):W396-400. doi:10.2214/AJR.09.3171

124. Kang TW, Lee MW, Song KD, et al. Ultrasound-guided radiofrequency ablation using a new electrode with an electromagnetic position sensor for hepatic tumors difficult to place an electrode: a Preliminary Clinical Study. Cardiovasc Intervent Radiol. 2017;40(12):1891-1898. doi:10.1007/s00270-017-1751-9

125. Nishigaki Y, Hayashi $H$, Tomita $E$, et al. Usefulness of contrast-enhanced ultrasonography using Sonazoid for the assessment of therapeutic response to percutaneous radiofrequency ablation for hepatocellular carcinoma. Hepatol Res. 2015;45 (4):432-440. doi:10.1111/hepr.12370

126. Kudo M, Ueshima K, Osaki Y, et al. B-mode ultrasonography versus contrast-enhanced ultrasonography for surveillance of hepatocellular carcinoma: a Prospective Multicenter Randomized Controlled Trial. Liver Cancer. 2019;8(4):271-280. doi:10.1159/000501082

127. Meloni MF, Francica G, Chiang J, Coltorti A, Danzi R, Laeseke PF. Use of contrast-enhanced ultrasound in ablation therapy of HCC: planning, guiding, and assessing treatment response. J Ultrasound Med. 2020. 
128. Bansal S, Gui J, Merrill C, Wong JK, Burak KW, Wilson SR. Contrast-enhanced US in local ablative therapy and secondary surveillance for hepatocellular carcinoma. Radiographics. 2019;39(5):1302-1322. doi:10.1148/rg.2019180205

129. Song KD, Lee MW, Rhim H, et al. Percutaneous US/MRI fusion-guided radiofrequency ablation for recurrent subcentimeter hepatocellular carcinoma: technical feasibility and therapeutic outcomes. Radiology. 2018;288(3):878-886. doi:10.1148/ radiol.2018172743

130. Sala M, Llovet JM, Vilana R, et al. Initial response to percutaneous ablation predicts survival in patients with hepatocellular carcinoma. Hepatology. 2004;40(6):1352-1360. doi:10.1002/ hep. 20465

131. Takahashi S, Kudo M, Chung H, et al. Initial treatment response is essential to improve survival in patients with hepatocellular carcinoma who underwent curative radiofrequency ablation therapy. Oncology. 2007;72(Suppl 1):98-103. doi:10.1159/ 000111714

132. Solbiati L. New applications of ultrasonography: interventional ultrasound. Eur J Radiol. 1998;27(Suppl 2):S200-6. doi:10.1016/ S0720-048X(98)00063-1

133. Clasen S, Rempp H, Hoffmann R, Graf H, Pereira PL, Claussen CD. Image-guided radiofrequency ablation of hepatocellular carcinoma (HCC): is MR guidance more effective than CT guidance? Eur J Radiol. 2014;83(1):111-116. doi:10.1016/j. ejrad.2013.09.018

134. Di Martino M, Marin D, Guerrisi A, et al. Intraindividual comparison of gadoxetate disodium-enhanced MR imaging and 64-section multidetector CT in the Detection of hepatocellular carcinoma in patients with cirrhosis. Radiology. 2010;256 (3):806-816. doi:10.1148/radiol.10091334

135. Rempp H, Loh H, Hoffmann R, et al. Liver lesion conspicuity during real-time MR-guided radiofrequency applicator placement using spoiled gradient echo and balanced steady-state free precession imaging. J Magn Reson Imaging. 2014;40(2):432-439. doi:10.1002/jmri.24371

136. Hoffmann R, Rempp H, Kessler DE, et al. MR-guided microwave ablation in hepatic tumours: initial results in clinical routine. Eur Radiol. 2017;27(4):1467-1476. doi:10.1007/s00330-016-4517-x

137. Weiss J, Winkelmann MT, Gohla G, et al. MR-guided microwave ablation in hepatic malignancies: clinical experiences from 50 procedures. Int $J$ Hyperthermia. 2020;37(1):349-355. doi:10.1080/02656736.2020.1750713

138. Ahn SJ, Lee JM, Lee DH, et al. Real-time US-CT/MR fusion imaging for percutaneous radiofrequency ablation of hepatocellular carcinoma. J Hepatol. 2017;66(2):347-354. doi:10.1016/j. jhep.2016.09.003

139. Lee MW, Rhim H, Cha DI, et al. Percutaneous radiofrequency ablation of hepatocellular carcinoma: fusion imaging guidance for management of lesions with poor conspicuity at conventional sonography. AJR Am J Roentgenol. 2012;198(6):1438-1444. doi:10.2214/AJR.11.7568

140. Song KD, Lee MW, Rhim H, Cha DI, Chong Y, Lim HK. Fusion imaging-guided radiofrequency ablation for hepatocellular carcinomas not visible on conventional ultrasound. AJR Am J Roentgenol. 2013;201(5):1141-1147. doi:10.2214/AJR.13.10532

141. Lee MW, Rhim H, Cha DI, Kim YJ, Lim HK. Planning US for percutaneous radiofrequency ablation of small hepatocellular carcinomas $(1-3 \mathrm{~cm})$ : value of fusion imaging with conventional US and CT/MR images. J Vasc Interv Radiol. 2013;24(7):958-965. doi:10.1016/j.jvir.2013.04.007

142. Kunishi Y, Numata K, Morimoto M, et al. Efficacy of fusion imaging combining sonography and hepatobiliary phase MRI with Gd-EOB-DTPA to detect small hepatocellular carcinoma. AJR Am J Roentgenol. 2012;198(1):106-114. doi:10.2214/ AJR.10.6039
143. Minami Y, Chung H, Kudo M, et al. Radiofrequency ablation of hepatocellular carcinoma: value of virtual CT sonography with magnetic navigation. AJR Am J Roentgenol. 2008;190(6):W33541. doi:10.2214/AJR.07.3092

144. Lim S, Lee MW, Rhim $\mathrm{H}$, et al. Mistargeting after fusion imaging-guided percutaneous radiofrequency ablation of hepatocellular carcinomas. J Vasc Interv Radiol. 2014;25(2):307-314. doi:10.1016/j.jvir.2013.10.025

145. Kang TW, Lee MW, Choi SH, et al. A novel electrode with electromagnetic tip tracking in ultrasonography-guided radiofrequency ablation: a phantom, ex vivo, and in vivo experimental study. Invest Radiol. 2015;50(2):81-87. doi:10.1097/ RLI.0000000000000103

146. Lee MW. Fusion imaging of real-time ultrasonography with CT or MRI for hepatic intervention. Ultrasonography. 2014;33 (4):227-239. doi:10.14366/usg.14021

147. Bo XW, Xu HX, Guo LH, et al. Ablative safety margin depicted by fusion imaging with post-treatment contrast-enhanced ultrasound and pre-treatment CECT/CEMRI after radiofrequency ablation for liver cancers. $\mathrm{Br} \quad J$ Radiol. 2017;90 (1078):20170063. doi:10.1259/bjr.20170063

148. Li K, Su ZZ, Xu EJ, Ju JX, Meng XC, Zheng RQ. Improvement of ablative margins by the intraoperative use of CEUS-CT/MR image fusion in hepatocellular carcinoma. BMC Cancer. 2016;16:277. doi:10.1186/s12885-016-2306-1

149. Feng K, Yan J, Li X, et al. A randomized controlled trial of radiofrequency ablation and surgical resection in the treatment of small hepatocellular carcinoma. J Hepatol. 2012;57 (4):794-802. doi:10.1016/j.jhep.2012.05.007

150. Uhlig J, Sellers CM, Stein SM, Kim HS. Radiofrequency ablation versus surgical resection of hepatocellular carcinoma: contemporary treatment trends and outcomes from the United States National Cancer Database. Eur Radiol. 2019;29(5):2679-2689. doi:10.1007/s00330-018-5902-4

151. Izumi N, Hasegawa K, Nishioka Y, et al. A multicenter randomized controlled trial to evaluate the efficacy of surgery vs. radiofrequency ablation for small hepatocellular carcinoma (SURF trial). $J$ Clin Oncol. 2019;37(15_suppl):4002. doi:10.1200/JCO.2019.37.15_suppl.4002

152. Hocquelet A, Trillaud H, Frulio N, et al. Three-dimensional measurement of hepatocellular carcinoma ablation zones and margins for predicting local tumor progression. $J$ Vasc Interv Radiol. 2016;27(7):1038-45 e2. doi:10.1016/j.jvir.2016.02.031

153. Smolock AR, Cristescu MM, Hinshaw A, et al. Combination transarterial chemoembolization and microwave ablation improves local tumor control for 3- to 5-cm hepatocellular carcinoma when compared with transarterial chemoembolization alone. Abdom Radiol (NY). 2018;43(9):2497-2504. doi:10.1007/ s00261-018-1464-9

154. Nishikawa H, Inuzuka T, Takeda $\mathrm{H}$, et al. Percutaneous radiofrequency ablation therapy for hepatocellular carcinoma: a proposed new grading system for the ablative margin and prediction of local tumor progression and its validation. $J$ Gastroenterol. 2011;46(12):1418-1426. doi:10.1007/s00535011-0452-4

155. Morimoto M, Numata $\mathrm{K}$, Kondou M, Nozaki A, Morita S, Tanaka K. Midterm outcomes in patients with intermediate-sized hepatocellular carcinoma: a randomized controlled trial for determining the efficacy of radiofrequency ablation combined with transcatheter arterial chemoembolization. Cancer. 2010;116(23):5452-5460. doi:10.1002/cncr.25314

156. Liapi E, Geschwind JF. Medium-sized HCC: achieving effective local tumor control with combined chemoebolization and radiofrequency ablation. Ann Surg Oncol. 2011;18(6):1527-1528. doi:10.1245/s10434-011-1679-2 
157. Peng ZW, Zhang YJ, Liang HH, Lin XJ, Guo RP, Chen MS. Recurrent hepatocellular carcinoma treated with sequential transcatheter arterial chemoembolization and RF ablation versus RF ablation alone: a prospective randomized trial. Radiology. 2012;262(2):689-700. doi:10.1148/radiol.11110637

158. Shibata T, Isoda H, Hirokawa $\mathrm{Y}$, Arizono S, Shimada $\mathrm{K}$, Togashi K. Small hepatocellular carcinoma: is radiofrequency ablation combined with transcatheter arterial chemoembolization more effective than radiofrequency ablation alone for treatment? Radiology. 2009;252(3):905-913. doi:10.1148/ radiol.2523081676

159. Wang W, Shi J, Xie WF. Transarterial chemoembolization in combination with percutaneous ablation therapy in unresectable hepatocellular carcinoma: a meta-analysis. Liver Int. 2010;30 (5):741-749. doi:10.1111/j.1478-3231.2010.02221.x
160. Peng ZW, Zhang YJ, Chen MS, et al. Radiofrequency ablation with or without transcatheter arterial chemoembolization in the treatment of hepatocellular carcinoma: a prospective randomized trial. J Clin Oncol. 2013;31(4):426-432. doi:10.1200/ JCO.2012.42.9936

161. Liu $\mathrm{W}, \mathrm{Xu} \mathrm{H}$, Ying $\mathrm{X}$, et al. Radiofrequency ablation (RFA) combined with transcatheter arterial chemoembolization (TACE) for patients with medium-to-large hepatocellular carcinoma: a retrospective analysis of long-term outcome. Med Sci Monit. 2020;26:e923263. doi:10.12659/MSM.923263

162. Young S, Golzarian J. Locoregional therapies in the treatment of 3- to 5-cm hepatocellular carcinoma: critical review of the literature. AJR Am $J$ Roentgenol. 2020;215(1):223-234. doi:10.2214/AJR.19.22098

\section{Publish your work in this journal}

The Journal of Hepatocellular Carcinoma is an international, peerreviewed, open access journal that offers a platform for the dissemination and study of clinical, translational and basic research findings in this rapidly developing field. Development in areas including, but not limited to, epidemiology, vaccination, hepatitis therapy, pathology and molecular tumor classification and prognostication are all considered for publication. The manuscript management system is completely online and includes a very quick and fair peer-review system, which is all easy to use. Visit http://www.dovepress.com/ testimonials.php to read real quotes from published authors. 